\title{
27. RADIOLARIANS FROM THE MARIANA TROUGH AND TRENCH REGION: DEEP SEA DRILLING PROJECT LEG 601
}

\author{
Stanley A. Kling, ${ }^{2}$ Scripps Institution of Oceanography, University of California, La Jolla, California
}

\begin{abstract}
Cenozoic radiolarians were recovered from all Leg 60 sites. At Sites 453 through 457 in the Mariana Trough, radiolarians are preserved only in the late Quaternary (0.3-0.9 m.y. and younger). At Sites 458 and 459 in the fore-arc region, fairly complete Neogene sequences are found, with isolated Eocene occurrences. On the west wall of the Mariana Trench, at Site 460, well-preserved Quaternary assemblages are underlain by sparse Tertiary ones, and at Site 461 only Quaternary material was found in place. At Site 452 on the deep Pacific floor east of the Trench, sparse Quaternary assemblages were recovered.

Cretaceous radiolarians were found in place at Site 452 (Campanian), and are reworked into Cenozoic sediments at Sites 460 and 461 (exact ages indeterminate).
\end{abstract}

\section{INTRODUCTION}

On Leg 60 of the Deep Sea Drilling Project, sites were occupied along an east-west transect through the Mariana Trough and Trench to the deep ocean floor east of the Trench (Figure 1). Radiolarians were recovered at all sites. Preservation and abundance are generally good in the uppermost, late Quaternary sections of all sites.

At the Mariana Trough sites (Sites 453-457) no radiolarians older than late Quaternary were preserved. At two sites east of the arc (Site 458 and 459), well-preserved late Quaternary assemblages are underlain by poorly preserved early Quaternary and Pliocene, followed by moderately well preserved Miocene material and isolated occurrences of Eocene material.

At two sites (Sites 460 and 461) on the deep western wall of the Mariana Trench, radiolarians are abundant and well preserved only in the latest Quaternary near the sediment surface. Below that, sparse Cretaceous forms are admixed with sparse Quaternary assemblages. Some Cretaceous specimens are well preserved and others poorly preserved. The lower part of Hole 460 also produced latest Eocene assemblages.

At the single site (Site 452) on the ocean floor just east of the Mariana Trench, a thin section of Neogene clay with sparse radiolarians is underlain by Cretaceous radiolarian cherts. Cretaceous forms are poorly preserved but identifiable as an assemblage of Campanian age.

\section{METHODS OF INVESTIGATION}

Soft sediment samples from the cores were disaggregated according to standard methods (Riedel and Sanfilippo, 1977) and sieved on a mesh with $44-\mu \mathrm{m}$ openings. Strewn slides of the fraction $>44 \mu \mathrm{m}$ were permanently mounted under cover glasses and examined for biostratigraphically significant species. These were

\footnotetext{
${ }_{2}^{1}$ Initial Reports of the Deep Sea Drilling Project, Volume 60.

2 Present address: 416 Shore View Lane, Leucadia, Ca.
}

examined with a light microscope in transmitted light. Chert from Site 452 was processed with hydrofluoric acid, according to the method of Pessagno and Newport (1972). The specimens freed by this method were examined with a scanning electron microscope.

Occurrences of species are recorded as abundances of morphotypes according to the concepts of Riedel and Sanfilippo (1971). Where appropriate, morphotypic limits were interpreted as evolutionary transitions (Riedel and Sanfilippo, 1971). Both kinds of limits were used in establishing zonations to determine the ages of samples.

Abundances are recorded as visual estimates in the following categories, with abbreviations and quantitative approximations in parentheses: abundant (A, one or more specimens in each microscope field), common ( $\mathrm{C}$, one in each 2-10 fields), few ( $\mathrm{F}$, one in each 10-20 fields), rare ( $R, 3-10$ specimens per slide), very rare $(+$, 1-2 specimens per slide), and barren (B).

Preservation can be viewed as the condition of individual specimens or a guess as to the completeness of the total assemblage. These concepts are both important but may be contradictory as, for example, in an assemblage consisting of well-preserved individuals belonging to only a few species. Since the condition of individuals can be more objectively judged than the status of the assemblage, greater emphasis is placed on that aspect in this study. Thus " $G$ " is used for good preservation, meaning a diverse assemblage of generally well-preserved specimens; " $M$ " for assemblages of moderately well preserved individuals even though species diversity may be low; and " $P$ " for poorly preserved specimens of a few or many species. Completeness of assemblages, it may be noted, can also be judged from occurrence charts.

\section{BIOSTRATIGRAPHIC ZONATION}

Radiolarian zonations already established for tropical regions (Fig. 2) were used for Leg 60 material. For the Quaternary, the zones of Nigrini (1971) were used with the modifications of Johnson and Knoll (1975). For the Tertiary, the zonation devised by Riedel and 


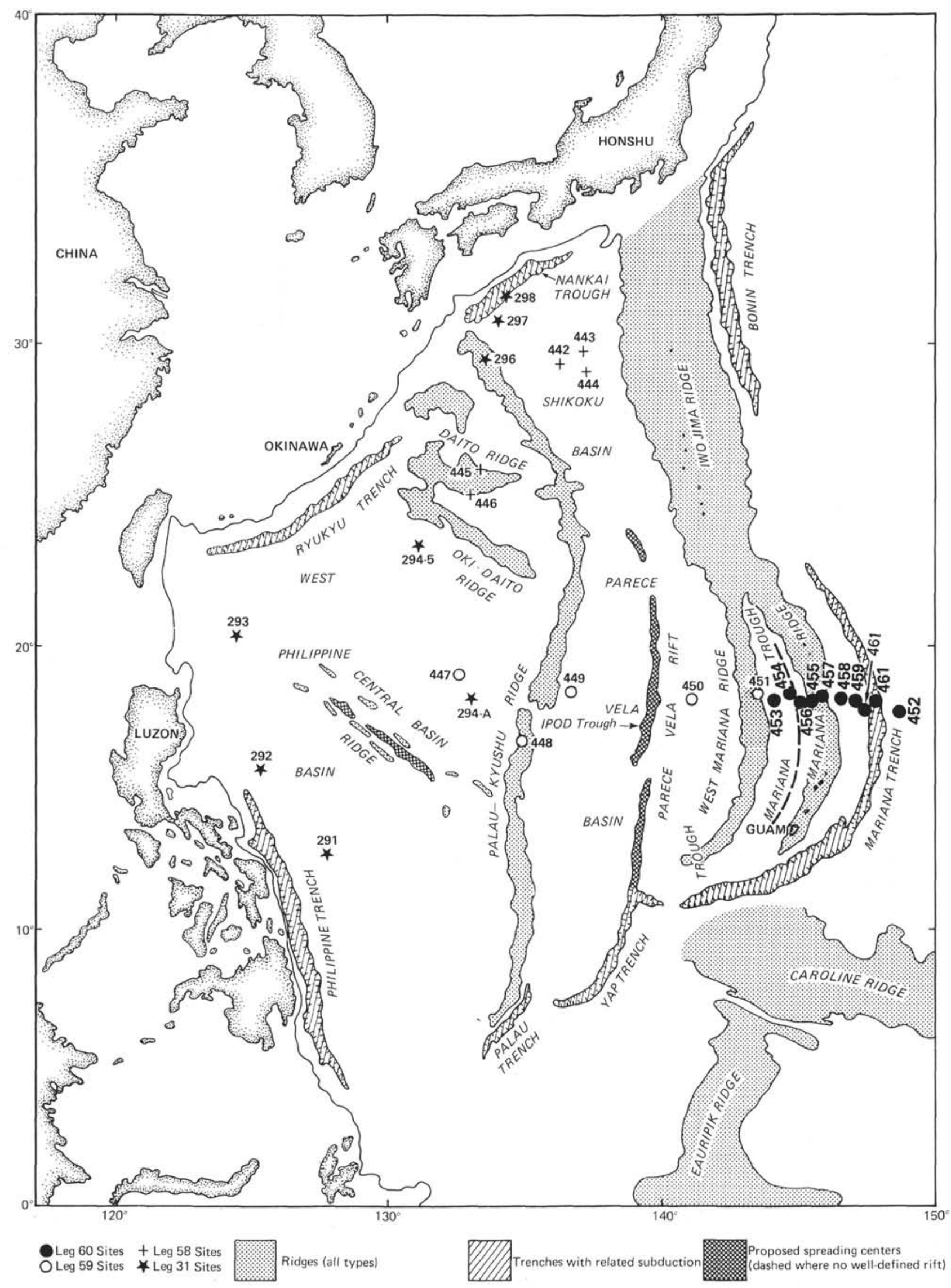

Figure 1. Index map showing locations of Leg 60 sites. 


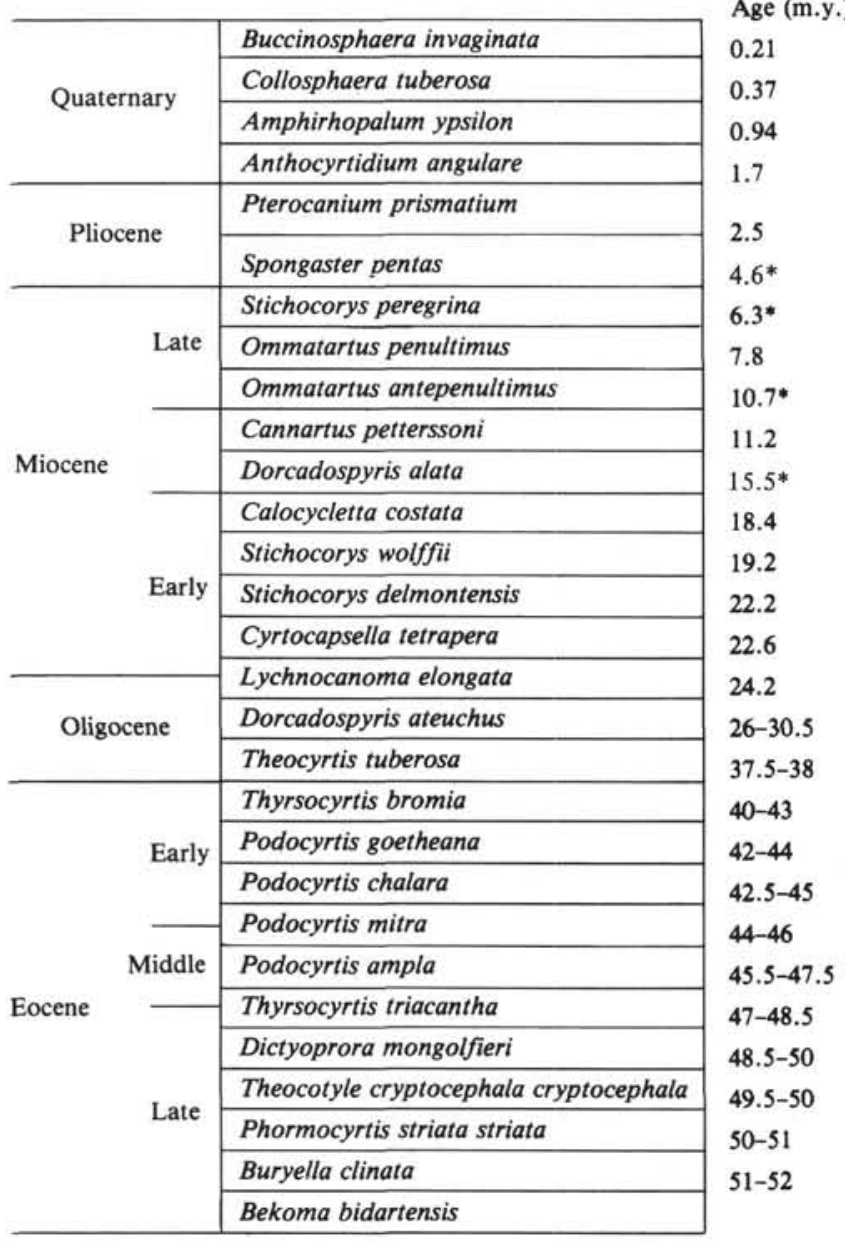

Figure 2. Chart of Cenozoic radiolarian zones and estimated absolute ages. Asterisks mark ages based on altered zone definitions. See text for explanation and sources.

Sanfilippo (1970) and later modified (Riedel and Sanfilippo, 1978) was used.

Absolute ages derived from direct comparisons of paleomagnetic and biostratigraphy have been estimated for the Quaternary zones by Johnson and Knoll (1975) and for the Neogene zones by Theyer, et al. (1978). It should be noted that some biostratigraphic events dated by Theyer et al. (1978) differ slightly from those used to define the zones of Riedel and Sanfilippo (1978). Less direct and therefore less precise estimates of the ages of Paleogene zones can be made from DSDP zone comparisons (e.g., Bukry et al., 1973; and Vincent, 1974) and from Hardenbol and Berggren (1978).

For the Cretaceous, the zonation of Riedel and Sanfilippo (1974) is most appropriate, but a correlation with the zones of Pessagno (1976) can also be made.

\section{RADIOLARIANS AT EACH SITE}

\section{Site 452}

$\left(17^{\circ} 40.19^{\prime} \mathrm{N}, 148^{\circ} 37.73^{\prime} \mathrm{E}\right.$, depth $\left.5858 \mathrm{~m}\right)$

Radiolarians of both Cenozoic and Cretaceous age were recovered from this site. Cenozoic forms occur in the brown clay unit where they are sparse and low in species diversity. No age-diagnostic forms were identifiable.

Cretaceous radiolarians were recovered from both cherts and associated mudstones at the bottom of the holes. Assemblages in both lithologies are essentially the same and can be placed in the Amphipyndax enesseffi Zone of Riedel and Sanfilippo (1974), of approximately Campanian age. Enough species of Pessagno's (1976) zones are present to indicate a correlation with his Campanian Crucella espartoensis Zone.

Site 453

$\left(17^{\circ} 54.42^{\prime} \mathrm{N}, 143^{\circ} 40.95^{\prime} \mathrm{E}\right.$, depth $\left.5862 \mathrm{~m}\right)$

Well-preserved radiolarians occur only in the first four of 64 cores recovered from Hole 453. In addition, some badly altered (to an unidentifed isotropic mineral) specimens were recovered from Core 17 , but no species could be identified.

Species upon which Nigrini's (1971) zonation is based are very sparse but the latest Quaternary Buccinosphaera invaginata Zone can be recognized in Core 1, the next older Collosphaera tuberosa Zone in Cores 1 and 2, and the Amphirhopalum ypsilon Zone in Cores 2 and 3. Preservation of biogenic silica thus extends to a sediment depth of about 32 meters or about halfway through the Quaternary (0.37-0.94 m.y.).

\section{Site 454 \\ $\left(18^{\circ} 00.78^{\prime} \mathrm{N}, 144^{\circ} 31.92^{\prime} \mathrm{E}\right.$, depth $\left.3818 \mathrm{~m}\right)$}

Well-preserved radiolarians are generally abundant in the five cores from Hole 454 and the first four of 16 cores from Hole 454A. Zone-diagnostic species are very rare, but the latest Quaternary Buccinosphaera invaginata Zone can be recognized in Core 1 of Hole 454, the Collosphaera tuberosa Zone in Core 3 of Hole 454 and Core 1 of Hole 454A.

Biogenic silica is preserved through the 38.5 meters of Hole 454, reaching the Amphirhopalum ypsilon Zone (0.37-0.9 m.y.). Deeper occurrences in Hole 454A are in core catcher samples only and likely represent contamination.

\section{Site 455 \\ $\left(17^{\circ} 51.26^{\prime} \mathrm{N}, 1^{\circ} 5^{\circ} 21.48^{\prime} \mathrm{E}\right.$, depth $\left.3468 \mathrm{~m}\right)$}

Radiolarians are well represented in Cores 1, 2, and 3 , rare in Cores 4 through 9 , and lacking in Cores 10 and 11. The latest Quaternary Buccinosphaera invaginata Zone is represented in Cores 1 and 2, and the Collosphaera tuberosa Zone in Core 2. No older zones are recognizable.

The record of abundant biogenic silica extends back through the mid-Quaternary $(0.37-0.94$ m.y. $)$ at a sediment depth of 86 meters.

\section{Site 456}

$\left(17^{\circ} 54.68\right.$ ' $\mathrm{N}, 145^{\circ} 10.77^{\prime} \mathrm{E}$, depth $\left.3590 \mathrm{~m}\right)$

Radiolarians are well preserved in late Quaternary cores from this site. Although zone-defining species are very scarce, the Buccinosphaera invaginata Zone can be 
recognized in Cores 1, 2 and 3 of Hole 456 and in Core 1 of Hole 456A. The Collosphaera tuberosa Zone is represented in Core 4 of Hole 456, and Core 1 of Hole 456A.

Biogenic silica is not preserved in sediment older than mid-Quaternary (0.37-0.94 m.y.) or deeper in the sediment than 53 meters.

\section{Site 457 \\ $\left(17^{\circ} 49.99^{\prime} \mathrm{N}, 145^{\circ} 49.02^{\prime} \mathrm{E}\right.$, depth $\left.2637 \mathrm{~m}\right)$}

Only a few radiolarian specimens were recovered from Site 457 where principally coarse, sandy sediment was cored. No age-diagnostic species were identified.

\section{Hole 458}

$\left(17^{\circ} 51.84^{\prime} \mathrm{N}, 146^{\circ} 56.06^{\prime} \mathrm{E}\right.$, depth $\left.3449 \mathrm{~m}\right)$

Radiolarians ranging from Quaternary through Oligocene ages occur in cores from this hole. Preservation is poor in the Pliocene, but otherwise generally good. Lack of several zone-defining species in the Miocene hinders exact placement of boundaries.

The Quaternary Collosphaera tuberosa Zone is recognizable in Core 1 . Core 2 is also Quaternary, but no zones can be recognized.

Core 3 is questionably Pliocene, but Core 4 contains no age-diagnostic species though it is dated Pliocene on the basis of calcareous nannofossils.

The late Miocene Ommatartus penultimus Zone is questionably recognized in Core 5. Apparently a hiatus spanning the latest Miocene (Stichocorys peregrina Zone) and possibly part of the early Pliocene occurs between Cores 3 and 5. The Ommatartus antepenultimus Zone is recognizable in Cores 5 and 6.

The middle Miocene Cannartus petterssoni Zone is questionably identified only in Cores 6 and 7 in samples lacking Dorcadospyris alata and with isolated single specimens of Ommatartus hughesi. In Cores 7 through 10 the Dorcadospyris alata Zone is tentatively recognized.

The early Miocene Calocycletta costata Zone is recognized in Cores 11 and 12 . Cores 13 and 14 belong to the Stichocorys wolffii Zone. A hiatus separates that zone from the Cyrtocapsella tetrapera Zone, with the intervening Stichocorys delmontensis Zone missing. The C. tetrapera Zone is identified in Cores 15 through 19.

No zones can be distinguished in Cores 21 and 22 which are either early Miocene or late Oligocene in age. The boundary between the early Oligocene Theocyrtis tuberosa and late Oligocene Dorcadospyris ateuchus zones occurs between Cores 22 and 24 .

One sample from Core 27 (Sample 27-1, 24-26 cm) contains the following well-preserved assemblages belonging to the early Eocene Buryella clinata Zone: Buryella clinata (C), Calocycloma castum (R), Ceratospyris articulata (R), Dictyoprora ovata (F), Lithochytris archaea (C), Lychnocanoma babylonis (R), Phormocyrtis striata exquisita (F), Podocyrtis diamesa (R),
Podocyrtis papalis (F), Theocotyle ficus (R), and Thyrsocyrtis hirsuta hirsuta (F).

\section{Site 459 \\ $\left(17^{\circ} 51.75^{\prime} \mathrm{N}, 147^{\circ} 18.09^{\prime} \mathrm{W}\right.$, depth $\left.4120 \mathrm{~m}\right)$}

Radiolarians occur through most of the sedimentary section cored at Site 459, interrupted however, by some barren intervals. Best preservation is during the Quaternary, the middle and early Miocene, and in the Eocene.

The latest Quaternary zones of Buccinosphaera invaginata and Collosphaera tuberosa are represented in Core 1 of Holes 459 and 459B. No other Quaternary zones are recognizable. In Hole 459B, radiolarians are sparse in Cores 2 and 3, Cores 4 and 5 are barren, and Core 6 contains rare radiolarians.

Miocene is represented in Hole 459B, with the late Miocene Ommatartus antepenultimus Zone recognized in Core 7 . Core 8 is only sparsely fossiliferous, and Cores 9 through 14 are barren.

Core 15, in Hole 459B, contains curved, flat spines of the genus Oroscena, which belong to the unusual orosphaerid group (Friend and Riedel, 1967) and have so far been reported only from the middle Miocene Cannartus petterssoni Zone (Kling, 1971). Core 20 is also interpreted as belonging to the Cannartus petterssoni Zone. The Dorcadospyris alata Zone can be recognized in Core 21. Cores 22 through 24 represent either the same zone or the next older Calocycletta costata Zone, which is clearly recognizable in Cores 24 and 25 .

The lower Miocene Stichocorys wolffii Zone is represented in Cores 25 through 27 . No other Neogene zones can be identified.

Two Eocene zones can be identified on the basis of well-preserved assemblages near the bottom of Hole 459B. In Core 58, the latest Eocene Thyrsocyrtis bromia Zone is represented by the following assemblage: Calocyclas turris (R), Dictyoprora mongolieri (R), Eusyringium fistuligerum (R), Lychnocanoma babylonis (+), Theosphaerella ptomatus (R), Thyrsocyrtis rhizodon (F), T. tetracantha $(+)$, and Tristylospyris triceros $(?)(+)$. The Podocyrtis chalara Zone, of late Eocene age but not contiguous with the $T$. bromia Zone, is recognized in Core 59 on the basis of the following assemblage: Calocycloma castum (R), Calocyclas hispida (R), Cryptoprora ornata (R), Dictyoprora mongolfieri (C), Eusyringium fistuligerum (R), Lithochytris verspertilio (R), Lithocyclia ocellus (R), Periphaena decora (F), Podocyrtis chalara (F), Podocyrtis papalis (R), Rhopalocanium ornatum (R), Thecosphaerella ptomatus (R), Theocotyle ficus (R), Thyrsocyrtis rhizodon (F), Thyrsocyrtis triacantha (R).

\section{Site 460 \\ $\left(17^{\circ} 40.14^{\prime} \mathrm{N}, 147^{\circ} 35.92^{\prime} \mathrm{E}\right.$, depth $\left.6450 \mathrm{~m}\right)$}

In the two holes drilled at Site 460 , well-preserved Quaternary assemblages are underlain by sparse Ter- 
tiary ones which contain reworked Cretaceous specimens.

The only Quaternary zone recognizable is the $B u c$ cinosphaera invaginata Zone, in Core 1 of Holes 460 and $460 \mathrm{~A}$.

Reworked early Oligocene (unzoned) and late Eocene (unzoned) can be recognized in the Quaternary assemblages (unzoned) of Cores 3 and 4 of Hole 460A, and Core 5 contains sparse Quaternary assemblages.

Eocene assemblages belonging to the Thrysocyrtis bromia Zone are recognizable in Cores 5 through 8 of Hole 460 . Older species within the sequence (within the same zone) suggest a normal sequence through these cores.

Cretaceous radiolarians are sparse in Core 1 of Hole 460 and in Cores 2 through 5 of Hole 460A. In the aggregate, species representative of the Amphipyndax enesseffi Zone are present, but many of the species are unidentifiable and probably represent other ages as well. This is supported by different states of preservation; some specimens are still of opaline composition, whereas others have been recrystallized to quartz.

\section{Site 461 \\ $\left(17^{\circ} 46.05^{\prime} \mathrm{N}, 147^{\circ} 41.18\right.$ ' , depth $\left.7029 \mathrm{~m}\right)$}

Both holes cored at Site 461 contain Quaternary radiolarians with reworked Cretaceous specimens. Only one zone, the latest Quaternary Buccinosphaera invaginata Zone can be recognized, in Core 1 of both Holes 461 and $461 \mathrm{~A}$.

Cretaceous radiolarians are admixed through both holes, but no zone-diagnostic assemblages can be identified. Preservation both as opal and as recrystallized quartz indicate various source horizons for the Cretaceous specimens.

\section{LIST OF CENOZOIC SPECIES}

Cenozoic species identified in this study are listed in alphabetical order with recent bibliographic references and references to appearances in tables of the present chapter.

Artophormis barbadensis (Ehrenberg)-Riedel and Sanfilippo, 1970, p. 532 , pl. 13 , fig. 5 .

This chapter: Table 5.

Artophormis gracilis Riedel, 1959, p. 300, pl. 2, figs. 12, 13.

This chapter: Table 5.

Amphirhopalum ypsilon Haeckel-Nigrini, 1967, p. 35, pl. 3, figs. 3a-d.

This chapter: Tables $3,4,6,7,8$.

Buccinosphaera invaginata Haeckel-Nigrini, 1971, p. 445 , pl. 34.1 , fig. 2 .

This chapter: Tables $1,2,3,4,6,7,8$.

Buryella clinata Foreman, 1973, p. 433, pl. 8, figs. 1-3, pl. 9, fig. 19.

Calocyclas hispida (Ehrenberg)-Foreman, 1973, p. 434, pl. 1, figs. 12-15, pl. 9, fig. 18.

Calocyclas turris Ehrenberg-Riedel and Sanfilippo, 1970, p. 529, pl. 13 , figs. 3,4 .

This chapter: Table 7.

Calocycletta costata (Riedel)-Riedel and Sanfilippo, 1970, p. 535, pl. 14 , fig. 12.

This chapter: Tables 5, 6 .

Calocycletta robusta Moore-Riedel and Sanfilippo, 1978, p. 66, pl. 3 , figs. 10,11 .

This chapter: Table 5

Calocycletta serrata Moore, 1972, p. 148, pl. 2, figs. 1-3.

This chapter: Table 5.
Table 1. Occurrences of selected Cenozoic radiolarian species, Site 453.

\begin{tabular}{|c|c|c|c|c|c|c|c|}
\hline & For legend, see text & Species & & & & & \\
\hline Age & $\begin{array}{l}\text { Radiolarian } \\
\text { Zone }\end{array}$ & $\begin{array}{c}\text { Core-Section } \\
\text { (interval in cm) }\end{array}$ & 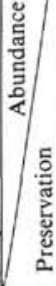 & 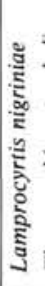 & 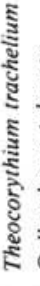 & 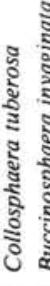 & 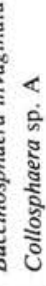 \\
\hline \multirow{5}{*}{ Quaternary } & Buccinosphaera invaginata & $\begin{array}{l}1-1,70-72 \\
1-2,70-72 \\
1-3,70-72 \\
1-4,70-72 \\
1-5,70-72\end{array}$ & $\begin{array}{l}\text { CG } \\
\text { CG } \\
\text { CG } \\
\text { AG } \\
\text { AG }\end{array}$ & $\begin{array}{l}\mathrm{R} \\
\mathrm{R} \\
\mathrm{R} \\
\mathrm{R}\end{array}$ & $\begin{array}{l}F \\
F \\
F \\
F\end{array}$ & $\begin{array}{lll}R & \\
R & \\
& \\
R & F \\
R & F\end{array}$ & $\begin{array}{l}2 \\
k \\
k\end{array}$ \\
\hline & Collosphaera tuberosa & $\begin{array}{l}1-6,70-72 \\
1, \mathrm{CC} \\
2-1,70-72 \\
2-2,70-72 \\
2-3,70-72\end{array}$ & $\begin{array}{l}\mathrm{AG} \\
\mathrm{AG} \\
\mathrm{AG} \\
\mathrm{AG} \\
\mathrm{CG}\end{array}$ & $\begin{array}{l}\mathrm{R} \\
\mathrm{R} \\
\mathrm{R} \\
\mathrm{R} \\
\mathrm{F}\end{array}$ & $\begin{array}{l}\mathrm{F} \\
\mathrm{R} \\
\mathrm{F} \\
\mathrm{F}\end{array}$ & $\begin{array}{l}R \\
R \\
R \\
R\end{array}$ & $\begin{array}{l}\mathrm{F} \\
\mathrm{R} \\
\mathrm{R} \\
\mathrm{F}\end{array}$ \\
\hline & Amphirhopalum ypsilon & $\begin{array}{l}2-4,70-72 \\
2, \mathrm{CC} \\
3-1,70-72 \\
3-2,70-72 \\
3, \mathrm{CC}\end{array}$ & $\begin{array}{l}\text { CG } \\
\text { CG } \\
\text { AG } \\
\text { CG } \\
\text { RM }\end{array}$ & $\begin{array}{l}\mathrm{R} \\
\mathrm{R} \\
\mathrm{R} \\
\mathrm{R}\end{array}$ & $\begin{array}{l}\mathrm{R} \\
\mathrm{R} \\
\mathrm{F} \\
\mathrm{R}\end{array}$ & & $\begin{array}{l}R \\
R \\
F\end{array}$ \\
\hline & \multirow[t]{2}{*}{ ? } & $\begin{array}{l}4-1,70-72 \\
4-2,70-72 \\
4-3,70-72 \\
4-4,70-72 \\
4-5,70-72\end{array}$ & $\begin{array}{c}\text { RM } \\
\text { RM } \\
\text { RM } \\
\text { B } \\
\text { B }\end{array}$ & \multirow{2}{*}{\multicolumn{3}{|c|}{$\mathrm{R}$}} & \\
\hline & & $\begin{array}{l}4-6,70-72 \\
4, C C\end{array}$ & $\begin{array}{l}\text { B } \\
\text { B }\end{array}$ & & & & \\
\hline
\end{tabular}

Calocycletta virginis (Haeckel)-Moore, 1972, p. 147, pl. 1, fig. 7. This chapter: Tables 5,6 .

Calocycloma castum (Haeckel)-Foreman, 1973, p. 434, pl. 1, figs. 7, $8,10$.

Cannartus laticonus Riedel, 1959, p. 291, pl. 1, fig. 5.

This chapter: Tables 5,6 .

Cannartus mammifer (Haeckel)-Riedel, 1959, p. 291, pl. 1, fig. 4. This chapter: Table 5.

Cannartus petterssoni Riedel and Sanfilippo, 1970, p. 520, p. 14, fig.

This chapter: Table 5 .

Cannartus prismaticus (Haeckel)-Riedel, 1959, p. 287, pl. 1, fig. 1, This chapter: Tables 5, 6.

Cannartus tubarius (Haeckel)-Riedel, 1959, p. 289, pl. 1, fig. 2. This chapter: Tables 5,6 .

Cannartus violina Haeckel-Riedel, 1959, p. 290, pl. 1, fig. 3. This chapter: Tables 5, 6 .

Carpocanopsis bramlettei Riedel and Sanfilippo, 1971, p. 1597, pl. $2 \mathrm{G}$, figs. 8-14; pl. 8, fig. 7 .

This chapter: Tables 5,6 .

Carpocanopsis cingulata Riedel and Sanfilippo, 1971, p. 1597, pl. 2G, figs. 17-21; pl. 8, fig. 8 . This chapter: Tables 5,6 .

Carpocanopsis cristata (Carnevale)-Riedel and Sanfilippo, 1971, p. 1597 , pl. $1 \mathrm{G}$, fig. 16 ; pl. $2 \mathrm{G}$, figs. 1-7.

This chapter: Table 6.

Carpocanopsis favosa (Haeckel)-Riedel and Sanfilippo, 1971, p. 1597, pl. 2G, figs. 15, 16; pl. 8, figs. 9-11.

This chapter: Table 5.

Ceratospyris articulata Ehrenberg-Sanfilippo and Riedel, 1973, p. 526 , pl. 15 , figs. $1-3$; pl. 31 , figs. 8,9 .

Collosphaera tuberosa Haeckel-Nigrini, 1971, p. 445 , pl. 34.1 , fig. 1.

This chapter: Tables 1, 2, 3, 4, 5, 6, 7, 8 .

Collosphaera sp. $A$ of Knoll and Johnson, 1975, p. 63, pl. 1, figs. 2, 7; pl. 2, figs. 4-6.

This chapter: Tables 1, 2, 3, 4, 5, 6, 7, 8 . 
Table 2. Occurrences of selected Cenozoic radiolarian species, Site 454.

\begin{tabular}{|c|c|c|c|c|c|c|c|}
\hline & For legend, see text & Species & & & & & \\
\hline Age & $\begin{array}{l}\text { Radiolarian } \\
\text { Zone }\end{array}$ & $\begin{array}{c}\text { Core-Section } \\
\text { (interval in } \mathrm{cm} \text { ) }\end{array}$ & 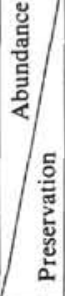 & 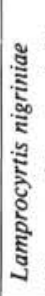 & 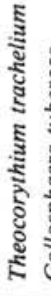 & 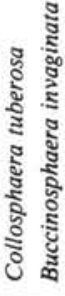 & 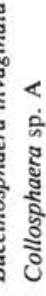 \\
\hline & B. invaginata & Hole 454 & $A G$ & P & & & \\
\hline & Collosphaera tuberosa & $\begin{array}{l}3-1,70-72 \\
3-2,70-72 \\
3-3,70-72 \\
3-4,70-72\end{array}$ & $\begin{array}{l}\mathrm{AG} \\
\mathrm{AG} \\
\mathrm{AG} \\
\mathrm{AG}\end{array}$ & $\begin{array}{l}\mathrm{R} \\
\mathrm{R} \\
\mathrm{R}\end{array}$ & $\begin{array}{l}F \\
F \\
F \\
F\end{array}$ & $\begin{array}{l}R \\
R \\
F \\
R\end{array}$ & $\begin{array}{l}\mathrm{F} \\
\mathrm{F} \\
\mathrm{R} \\
\mathrm{R}\end{array}$ \\
\hline & & $3, \mathrm{CC}$ & FM & $\mathrm{R}$ & $\mathbf{R}$ & $\mathrm{R}$ & $\mathbf{R}$ \\
\hline & ? & $\begin{array}{l}4-1,70-72 \\
4-2,70-72 \\
4-3,70-72 \\
4-4,70-72\end{array}$ & $\begin{array}{l}\mathrm{AM} \\
\mathrm{AM} \\
\mathrm{AM} \\
\mathrm{CM}\end{array}$ & $\begin{array}{l}+ \\
\mathrm{R} \\
\mathrm{R}\end{array}$ & $\begin{array}{l}F \\
R \\
R \\
F\end{array}$ & & \\
\hline Quaternary & & $\begin{array}{l}4-5,70-72 \\
4, C C \\
5, C C\end{array}$ & $\begin{array}{l}\mathrm{AM} \\
\mathrm{CG} \\
\mathrm{CG}\end{array}$ & $\begin{array}{l}\mathrm{R} \\
\mathrm{R}\end{array}$ & $\begin{array}{l}\mathrm{R} \\
\mathrm{R}\end{array}$ & R & \\
\hline & ? & $\begin{array}{l}\text { Hole 454A } \\
1-1,70-72 \\
1-2,70-72\end{array}$ & $\begin{array}{l}\mathrm{CM} \\
\mathrm{AG}\end{array}$ & $\begin{array}{l}\mathrm{R} \\
\mathrm{R}\end{array}$ & $\begin{array}{l}F \\
F\end{array}$ & $\begin{array}{l}\mathrm{R} \\
\mathrm{R}+\end{array}$ & + \\
\hline & Collosphaera tuberosa & $\begin{array}{l}1-3,70-72 \\
1-4,70-72\end{array}$ & $\begin{array}{l}\mathrm{AG} \\
\mathrm{CM}\end{array}$ & & & $\begin{array}{l}\mathrm{R} \\
\mathrm{R}\end{array}$ & $\begin{array}{l}\mathrm{R} \\
\mathrm{R}\end{array}$ \\
\hline & ? & $\begin{array}{l}1, C C \\
2-2,70-72 \\
2-3,106-108 \\
2, C C \\
3-3,52-54\end{array}$ & $\begin{array}{c}\mathrm{AG} \\
\mathrm{B} \\
\mathrm{RM} \\
\mathrm{B} \\
\mathrm{B}\end{array}$ & $\mathrm{R}$ & $\mathrm{F}$ & & + \\
\hline & ? & $\begin{array}{l}3, \mathrm{CC} \\
4-2,77-79 \\
4, \mathrm{CC}\end{array}$ & $\begin{array}{c}\text { FM } \\
\text { B } \\
\text { RM }\end{array}$ & $\mathbf{R}$ & & & \\
\hline
\end{tabular}

Table 3. Occurrences of selected Cenozoic radiolarian species, Site 455 .

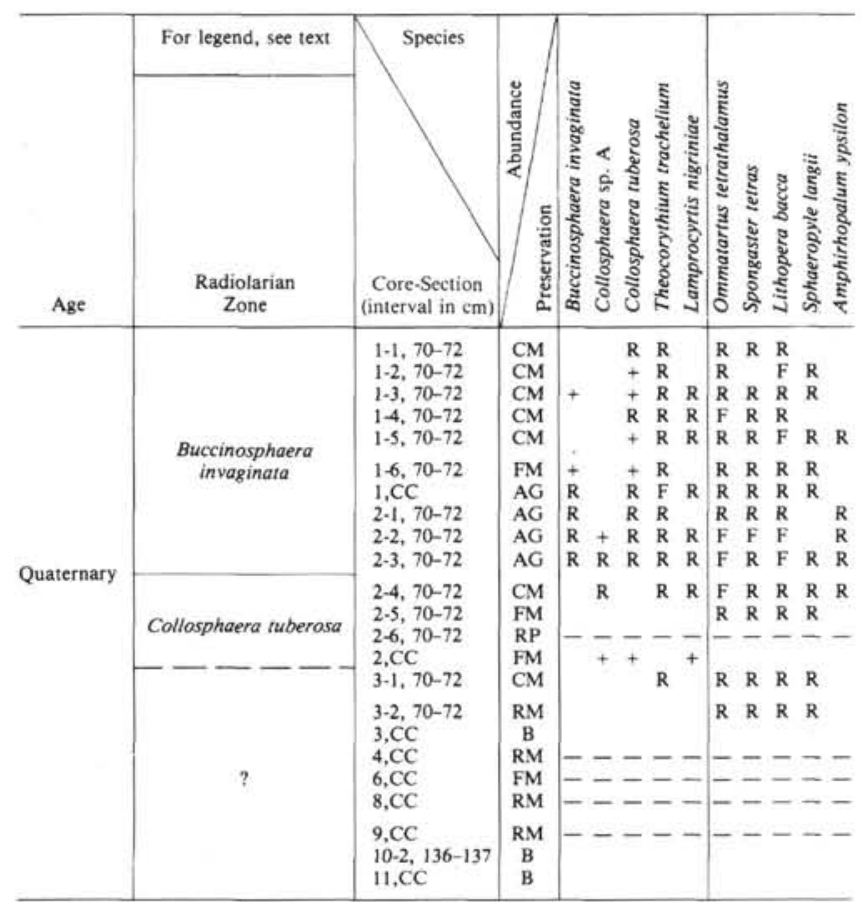

Table 4. Occurrences of selected Cenozoic radiolarian species, Holes 456 and 456A.

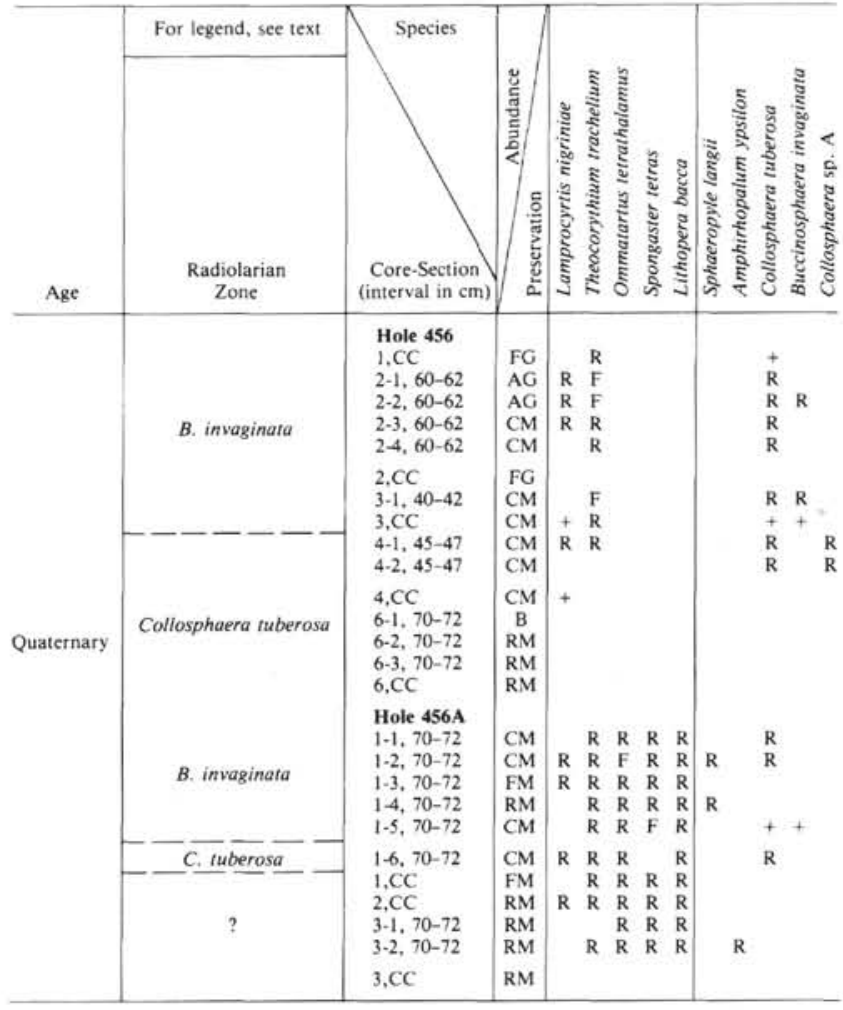

Cryptoprora ornata Ehrenberg-Riedel and Sanfilippo, 1971, p. 1597 , pl. 3D, figs. 10, 11.

Cyrtocapsella cornuta (Haeckel)-Sanfilippo and Riedel, 1970, p. 453 , pl. 1, figs. 19, 20.

This chapter: Tables 5, 6 .

Cyrtocapsella japonica (Nakaseko)-Sanfilippo and Riedel, 1970, p. 452 , pl. 1 , figs. $13-15$.

This chapter: Tables 5, 6 .

Cyrtocapsella tetrapera (Haeckel)-Sanfilippo and Riedel, 1970, p. 453 , pl. 1, figs. 16-18.

This chapter: Tables 5, 6 .

Dendrospyris bursa Sanfilippo and Riedel, 1973, p. 217, pl. 2, figs. 9-13.

This chapter: Table 6.

Dictyoprora armadillo (Ehrenberg)-Nigrini, 1977, p. 250, pl. 4, fig. 4 .

This chapter: Table 7.

Dictyoprora mongolfieri (Ehrenberg)-Nigrini, 1977, p. 250, pl. 4, fig. 7.

This chapter: Table 7.

Dictyoprora ovata (Haeckel)-Nigrini, 1977, p. 251, pl. 4, figs. 5, 6. This chapter: Table 7.

Dorcadospyris alata (Riedel), 1959, p. 293, pl. 1, figs. 11, 12. This chapter: Tables 5, 6 .

Dorcadospyris ateuchus (Ehrenberg)-Riedel and Sanfilippo, 1970, p. 523 , pl. 15 , fig. 4 .

This chapter: Table 5,6 .

Dorcadospyris dentata Haeckel-Riedel, 1957, p. 79, pl. 1, fig. 4. This chapter: Table 5.

Dorcadospyris simplex (Riedel), 1959, p. 293, pl. 1, fig. 10. This chapter: Tables 5, 6 .

Eucyrtidium yatsuoense Nakaseko, 1955, p. 110, pl. X, figs. 1a, b. This chapter: Table 6.

Eusyringium fistuligerum (Ehrenberg)-Foreman, 1973, p. 435, pl. 11 , fig. 6 .

Lamprocyrtis nigriniae (Caulet)-L. haysi Kling, 1973, p. 639, pl. 5, figs. 15,16 ; pl. 15, figs. 1-3.

This chapter: Tables $1,2,3,4,5,6,7,8$. 
Lamprocyrtis neoheteroporos Kling, 1973, p. 639, pl. 5, figs. 17, 18; pl. 15 , figs. 4,5 .

This chapter: Table 5.

Liriospyris stauropora (Haeckel)-Goll, 1968, p. 1431, pl. 175, figs. $1-3,7$; text-fig. 9 .

This chapter: Table 5 .

Lithochytris archaea Riedel and Sanfilippo, 1970, p. 528, pl. 9, fig. 7.

Lithochytris vespertilio Ehrenberg-Riedel and Sanfilippo, 1970, p. 528 , pl. 9 , figs. 8,9 .

Lithocyclia angusta (Riedel)-Riedel and Sanfilippo, 1970, p. 522, pl. 13, figs. $1,2$.

Lithocyclia aristotelis (Ehrenberg) group-Riedel and Sanfilippo, 1978, p. 70, pl. 6, fig. 6.

This chapter: Table 7.

Lithocyclia ocellus (Ehrenberg) group-Riedel and Sanfilippo, 1970, p. 522, pl. 5, figs. 1,2 .

Lithopera bacca Ehrenberg-Nigrini, 1967, p. 54, pl. 6, fig. 2.

This chapter: Tables $3,4,5,6,7,8$.

Lithopera baueri Sanfilippo and Riedel, 1970, p. 455, pl. 2, figs. 1, 2. This chapter: Table 5.

Lithopera neotera Sanfilippo and Riedel, 1970, p. 454, pl. 1, figs. 24-26, 28.

This chapter: Tables 5,6 .

Lithopera thornburgi Sanfilippo and Riedel, 1970, p. 455, pl. 2, figs. 4-6.

This chapter: Table 5 .

Lychnocanoma amphitrite Foreman, 1973, p. 437, pl. 11, fig. 10. This chapter: Table 7.

Lychnocanoma babylonis (Clark and Campbell) group-Riedel and Sanfilippo, 1970, p. 528, pl. 9, figs. 1-3.

This chapter: Table 7.

Ommatartus antepenultimus Riedel and Sanfilippo, 1970, p. 521, pl. 14, fig. 4.

This chapter: Tables 5, 6 .

Ommatartus hughesi Campbell and Clark-Riedel and Sanfilippo, 1978 , p. 71 , pl. 7 , fig. 7.

This chapter: Tables 5, 6 .

Ommatartus tetrathalamus (Haeckel)-Nigrini, 1967, p. 30, pl. 2 , figs. $4 a-d$.

This chapter: Tables $3,4,5,7,8$.

Oroscena sp. with curved, flat spines-Kling, 1971, p. 1086, pl. 3, fig. 7.

This chapter: Table 6.

Oroscena sp. with bifurcate spines-Kling, 1971, p. 1086, pl. 3, fig. 1 . This chapter: Table 6.

Periphaena decora Ehrenberg-Sanfilippo and Riedel, 1973, p. 523, pl. 8 , figs. $8-10$; pl. 27, figs. 2-5.

This chapter: Table 7.

Phormocyrtis striata exquisita (Koslova)-Foreman, 1973, p. 438, pl. 7 , figs. $1-4,7,8$; pl. 12 , fig. 5 .

Phormostichoartus corbula (Harting)-Nigrini, 1977, p. 252, pl. 1, fig. 10.

This chapter: Table 5 .

Phormostichoartus doliolum (Riedel and Sanfilippo)-Nigrini, 1977, p. 252, pl. 1, fig. 14 .

This chapter: Tables 5,6 .

Phormostichoartus marylandicus (Martin)-Nigrini, 1977, p. 253, pl. 2, figs. 1-3.

This chapter: Tables 5, 6 .

Podocyrtis chalara Riedel and Sanfilippo, 1970, p. 535, pl. 12, figs. 2, 3.

Podocyrtis diamesa Riedel and Sanfilippo-Sanfilippo and Riedel, 1973 , p. 531, pl. 20, figs. 9, 10; pl. 35, figs. $10,11$.

Podocyrtis papalis Ehrenberg-Riedel and Sanfilippo, 1970, p. 533, pl. 11 , fig. 1 .

Rhopalocanium ornatum Ehrenberg-Foreman, 1973, p. 439, pl. 2, figs. $8-10$; pl. 12 , fig. 3 .

Siphostichartus corona (Haeckel)-Nigrini, 1977, p. 257, pl. 2, figs. 5,6 .

This chapter: Tables 5, 6 .

Siphostichartus praecorona Nigrini, 1977, p. 258, pl. 2, figs. $8,9$. This chapter: Tables 5, 6 .

Sphaeropyle langii Dreyer-Foreman, 1975, p. 618, pl. 9, figs. 30, 31. This chapter: Tables 3, 4, 5, 6, 7, 8 .

Sphaeropyle robusta Kling-Foreman, 1975, p. 618, pl. 9, figs. 24-26. This chapter: Table 6.
Spongaster berminghami (Campbell and Clark)-Riedel and Sanfilippo, 1978, p. 73, pl. 2, figs. 14-16.

This chapter: Table 5 .

Spongaster tetras Ehrenberg-Riedel and Sanfilippo, 1971, p. 1589, pl. 1D, figs. 2-4.

This chapter: Tables 3, 4, 5, 6, 7, 8 .

Stichocorys armata (Haeckel)-Riedel and Sanfilippo, 1971, p. 1595, pl. 2E, figs. 13-15.

This chapter: Tables 5, 6 .

Stichocorys delmontensis (Campbell and Clark)-Sanfilippo and Riedel, 1970, p. 451, pl. 1, fig. 9.

This chapter: Tables 5, 6 .

Stichocorys diploconus (Haeckel)-Sanfilippo and Riedel, 1970, p. 451 , pl. 1, figs. 31,32 .

This chapter: Table 5 .

Stichocorys peregrina (Riedel)-Sanfilippo and Riedel, 1970, p. 451, pl. 1, fig. 10.

This chapter: Tables 5, 6 .

Stichocorys wolffii Haeckel-Riedel, 1957, p. 92, pl. 4, figs. 6, 7. This chapter: Tables 5, 6.

Thecosphaerella ptomatus-Sanfilippo and Riedel, 1973, p. 521, pl. 3 , figs. $14-18$; pl. 26 , fig. 2 .

Theocorys acroria Foreman, 1973, p. 439, pl. 5, figs. 11-13; pl. 12, fig. 2 .

Theocorys spongoconum-Kling, 1971, p. 1087, pl. 5, fig. 6 . This chapter: Tables 5, 6 .

Theocorythium trachelium (Ehrenberg)-Nigrini, 1967, p. 77, pl. 8, figs. 1, 2; pl. 9, figs. 1, 2 .

This chapter: Tables $1,2,3,4,5,6,7,8$.

Theocotyle ficus (Ehrenberg)-Foreman, 1973, p. 441, pl. 4, figs. 16-20.

Theocyrtis tuberosa Riedel, 1959, p. 298, pl. 2, figs. 10, 11. This chapter: Table 5.

Theocyrtis sp. aff. T. tuberosa Riedel-Riedel and Sanfilippo, 1971, pl. 3D, figs. 16-18.

This chapter: Table 5.

Thyrsocyrtis bromia Ehrenberg-Riedel and Sanfilippo, 1971, p. 1596 , pl. 8 , fig. 6 .

This chapter: Tables 5,7

Thyrsocyrtis hirsuta hirsuta (Krasheninnikov)-Foreman, 1973, p. 441, pl. 3, figs. 3-8; pl. 12, fig. 15.

Thyrsocyrtis rhizodon Ehrenberg-Riedel and Sanfilippo, 1970, p. 525 , pl. 7 , figs. 6,7 .

This chapter: Table 7.

Thyrsocyrtis tetracantha (Ehrenberg)-Riedel and Sanfilippo, 1971, p. 1596.

Thyrsocyrtis triacantha (Ehrenberg)-Foreman, 1973, p. 442, pl. 12, figs. 9-11.

Tristylospyris triceros (Ehrenberg)-Riedel and Sanfilippo, 1978, p. 82 , pl. 10 , fig. 12 .

This chapter: Tables 5, 6, 7 .

\section{CRETACEOUS RADIOLARIANS}

Radiolarians of Cretaceous age occur at three sites of DSDP Leg 60: at Site 452 on the deep sea floor east of the Mariana Trench, and at Sites 460 and 461 on the western wall of the Mariana Trench. Specimens from Site 452 were found in place in cherts and overlying mudstones at the bottom of the hole and constitute a coherent, datable assemblage. Only isolated specimens, reworked into Cenozoic sediments, were recovered from Sites 460 and 461 . Many of these are undescribed forms, and precise dating of the sparse assemblages is not yet possible. None of the assemblages is preserved well enough or is complete enough to warrant full systematic treatment. Therefore, identifiable taxa are listed separately for each of the sites.

\section{Site $\mathbf{4 5 2}$}

Cretaceous radiolarians occur in cherts from the bottom of Hole 452A and as loose specimens derived from 
Table 5. Occurrences of selected Cenozoic radiolarian species, Site 458.

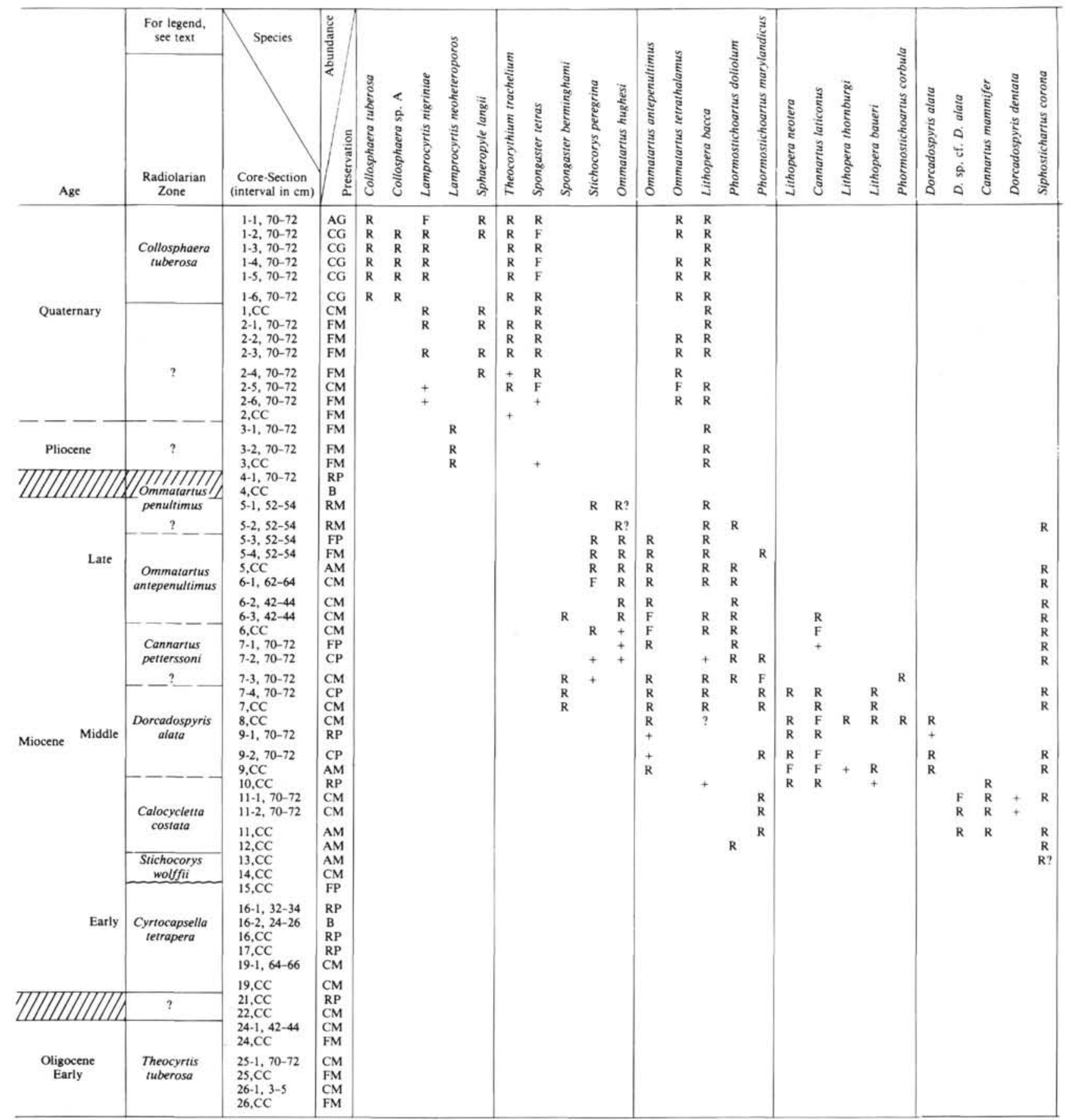

mudstone and porcellanite overlying the cherts and/or from drilling breccia of the chert itself. The specimens have been altered to and filled in with crystalline silica (quartz or chalcedony) and are generally poorly preserved. Nevertheless, through examination of both light and scanning electron microscopy (SEM), enough taxa can be identified to permit recognition of biostratigraphic zones.

The following samples contain significant quantities of Cretaceous radiolarians: Samples 452A-3-6, 20-22 $\mathrm{cm}$; 452A-3-6, 70-72 cm; 452A-5-1, 28-30 cm; and $452 \mathrm{~A}-5, \mathrm{CC}$
Material from two samples was examined in detail in the SEM-loose specimens from Sample 452A-5-1, $28-30 \mathrm{~cm}$ and specimens etched from cherts of Sample $452 \mathrm{~A}-5, \mathrm{CC}$ by means of hydrofluoric acid (Pessagno and Newport, 1972). The assemblages are similar, sharing the same major species, and represent the same zone. Amphipyndax enesseffi, Pseudoaulophacus pargueraensis, and Dictyomitra duodecimcostata duodecimcostata (included in D. torquata of Riedel and Sanfilippo, 1974) permit recognition of the $A$. enesseff $i$ Zone of Riedel and Sanfilippo (1974), assigned an age of "approximately Campanian" in European stage ter- 
Table 5. (Continued),

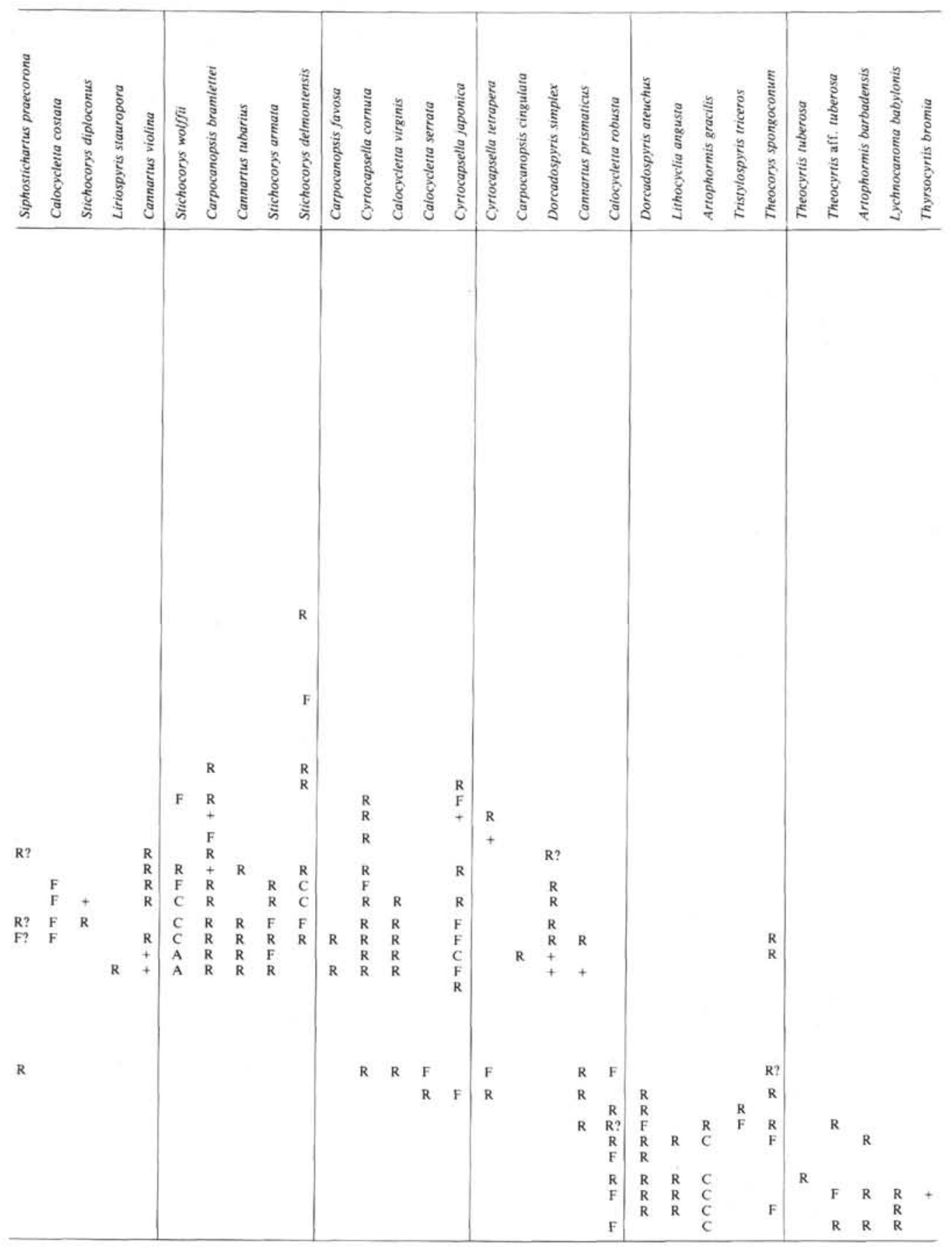

minology. The assemblage contains other species (listed below) reported from that zone and is apparently consistent with the emended definition of the $A$. enesseffi Zone proposed by Foreman (1977) for the Atlantic Ocean, though it is not known whether Amphipyndax tylotus, whose first appearance defines the next higher zone of the same name, occurs in the Pacific. The Site 452 assemblage contains few species in common with Campanian assemblages from California described by Pessagno (1976). Pending confirmation of the tentatively identified Phaseliforma laxa, it might be placed in the upper part of his Crucella espartoensis Zone. How- ever, the similarity of Dictyomitra duodecimcostata duodecimostata to Pessagno's (1976) D. formosa Squinabol suggests a position lower in that zone.

The following taxa characterize the Cretaceous assemblages. They are listed alphabetically with bibliographic references and citations of illustrations in this chapter.

\section{Acaeniotyle sp.}

This chapter: Plate 1, Figure 5. Actinommids gen. et. spp. indet.

This chapter: Plate 1, Figures 1-4.

Included in this category are several isolated specimens of spherical forms, some incompletely preserved, which are of uncertain 


\section{S. A. KLING}

Table 6. Occurrences of selected Cenozoic radiolarian species, Holes 459 and 459B.

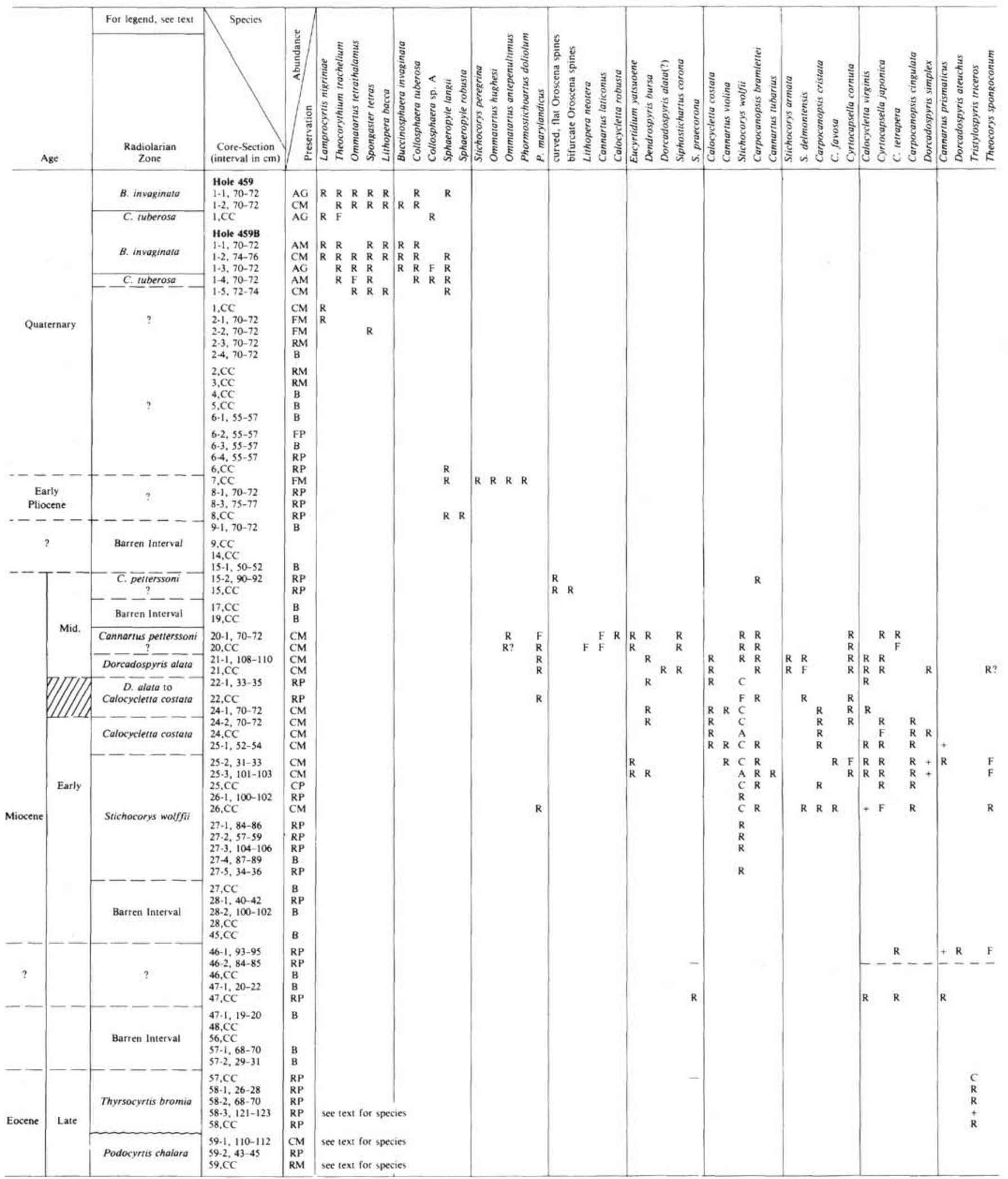


Table 7. Occurrences of selected Cenozoic radiolarian species, Site 460.

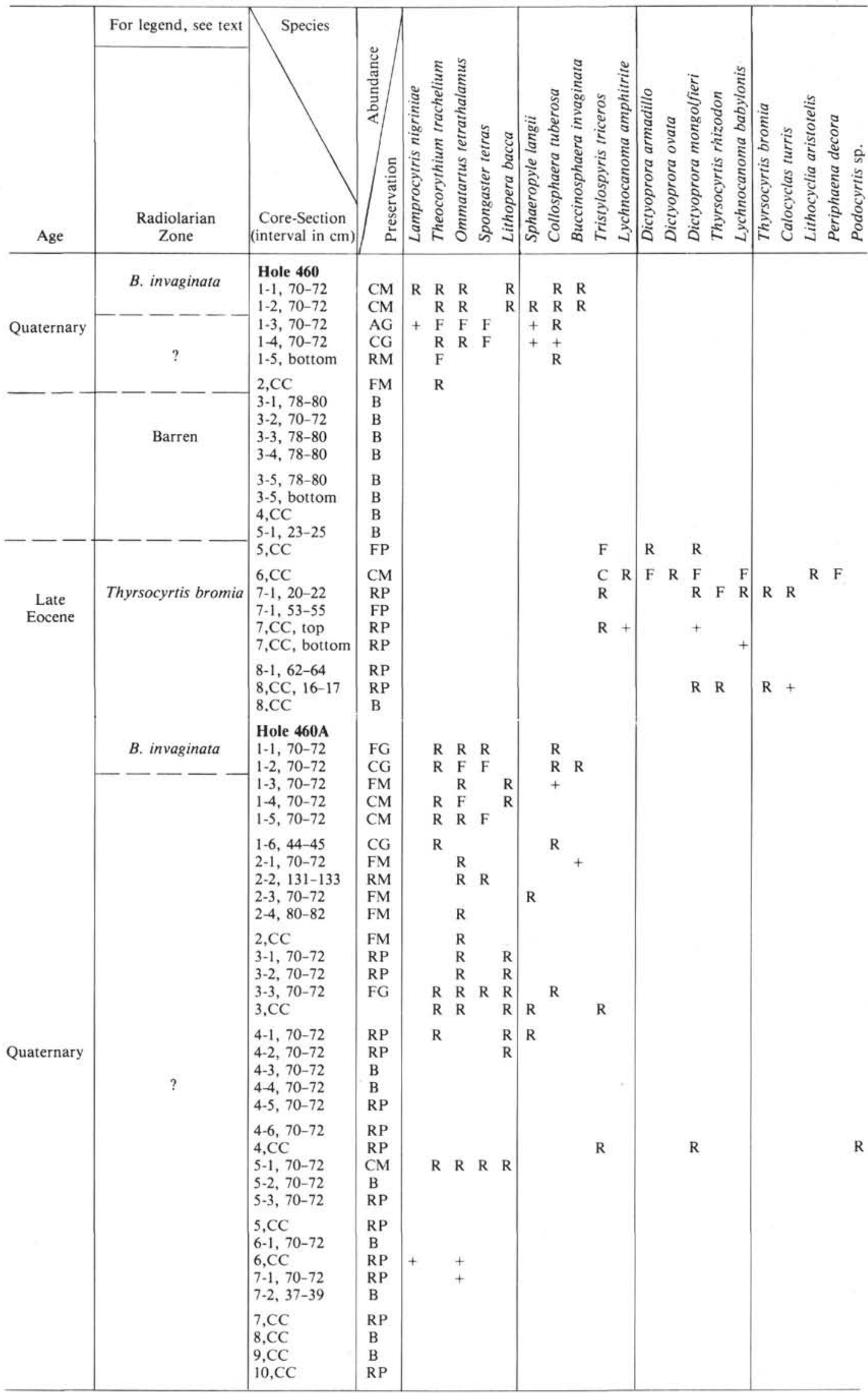


Table 8. Occurrences of selected Cenozoic radiolarian species, Site 461.

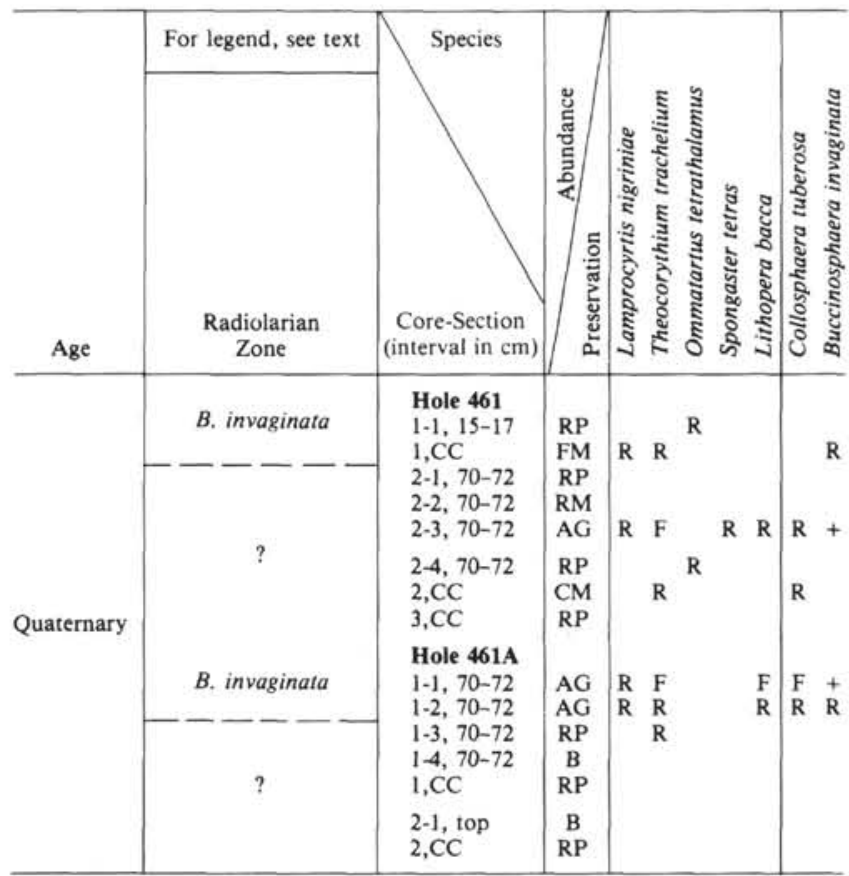

affinity to described species, but which may be restricted to the Mesozoic.

Afens liriodes Riedel and Sanfilippo, 1974, p. 775; pl. 11, fig. 11; pl. 13, figs. 14-16.

This chapter: Plate 1, Figures 23, 24; Plate 3, Figures 5, 6.

Alievium gallowayi (White)-Pessagno, 1972, p. 299, p. 25, figs. 4-6; pl. 26, fig. 5 ; pl. 31 , figs. 2,3 .

This chapter: Plate 1, Figures 15, 16(?)

Amphipyndax enesseffi Foreman, 1966, p. 356, text-figs. 10, 11. This chapter: Plate 2, Figure 12.

Amphipyndax stocki (Campbell and Clark), emend. Foreman, 1968, p. 78 , pl. 8 , figs. $12 \mathrm{a}-\mathrm{c}$.

This chapter: Plate 2, Figure 11.

Archaeodictyomitra sp.

This chapter: Plate 2, Figures 6(?), 7.

In this category are included multisegmented conical forms with strong costae that are not interrupted or constricted at the sutures between chambers.

Artostrobium urna Foreman, 1971, p. 1677, pl. 4, figs. 1, 2.

This chapter: Plate 1, Figure 20.

Clathropyrgus titthium Riedel and Sanfilippo, 1974, p. 775, pl. 3, fig.

12; pl. 12, figs. 9-12.

This chapter: Plate 1, Figure 21; Plate 3, Figure 7.

Cryptocapsular theoperids gen. et. spp. indet.

This chapter: Plate 2, Figures 16-19, 21-23, 24(?); Plate 3, Figure $8(?)$.

In this category are placed several unidentified forms sharing a large, generally spherical terminal chamber into which the cephalis and/or thorax are partially or wholly depressed.

Dictyomitra duodecimcostata duodecimcostata (Squinabol)-Foreman, 1978 , p. 746 , pl. 4 , figs. 8,9 .

This chapter: Plate 2, Figures 8, 9.

Dictyomitra koslovae Foreman, 1975, p. 614, pl. 7, fig. 4.

This chapter: Plate 2, Figure 10; Plate 3, Figure 4.

Dictyomitra spp.

This chapter: Plate 2, Figures 1-4.

In this category are included multisegmented conical forms with costae that are constricted, though not necessarily interrupted at intersegmental boundaries (sutures).

Dictyomitra(?) sp. X.

This chapter: Plate 2, Figure 5.

This distinctive form is apparently undescribed. The single specimen available for study is proximally conical, becoming inflated cylindrical distally. It bears numerous low costae ( 18 visible on the halfcircumference) some ( 3 visible) of which are extended proximally as vertical lamellae. The lamellae are fewer in number and relatively larger and the test broader and more finely costate than in the $D$. lamellicostata Foreman, 1968.

Hemicryptocapsa tuberosa Dumitrică(?), 1970, p. 71, pl. XII, fig. 78a; pl. XIII, figs. 78b-c, 79a-b; pl. XXI, fig. 135.

This chapter: Plate 2, Figure 20.

The form illustrated here resembles Dumitrică's species except for somewhat larger pores, but in the absence of internal structures positive identification cannot be made.

Patulibracchium sp.

This chapter: Plate 1, Figure 17.

Phaseliforma spp. cf. P. laxa Pessagno, 1972, p. 276, pl. 23, figs. 7-9.

This chapter: Plate 1, Figure 18.

The specimen illustrated here is similar in size, general shape, and surface texture to Pessagno's species, but it is somewhat less elongate and apparently less flattened.

Podocapsa(?) sp.

This chapter: Plate 1, Figure 22.

Pseudoaulophacus floresensis Pessagno(?), 1963, p. 200, pl. 2, figs. 2,5 ; pl. 4 , fig. 6 .

This chapter: Plate 1, Figure 11

The specimens available for study here are similar in size, shape, character of tholi, etc to Pessagno's species, but the lack of complete spines leaves the identification uncertain.

Pseudoaulophacus pargueraensis Pessagno, 1963, p. 204, pl. 2, figs. 4,$7 ;$ pl. 6 , figs. 4,5 .

This chapter: Plate 1, Figures 13, 14.

Pseudoaulophacus lenticulatus (White)(?)-Pessagno, 1963, p. 202, pl. 2, figs. 8-9.

This chapter: Plate 1, Figure 12.

The single specimen available for study here resembles Pessagno's material but most of the spines are apparently broken away and those that remain are somewhat narrower at the base.

Spongosaturnalis (?) spp.

This chapter: Plate 1, Figures 6, 7; Plate 3, Figures 1-3.

A number of fragments of spiny-ringed saturnalins occur in Leg 60 samples. Without more complete specimens they cannot be identified with certainty, and without cortical shells they cannot be placed in the genus Spongosaturnalis with certainty.

Stichomitra asymbatos Foreman group-Riedel and Sanfilippo, 1974, p. 780, pl. 10, figs. 1-7; pl. 15, fig. 5 .

This chapter: Plate 2, Figures 13, 14.

Theocampe salillum Foreman, 1971, pl. 4, fig. 5. This chapter: Plate 1, Figure 19.

\section{Site 460}

Cretaceous radiolarians occur in cores from Site 460 only as reworked specimens mixed with younger assemblages. Some are well preserved and others poorly preserved. This suggests a heterogeneous source for the reworked material with the implication that substantially different ages are involved in the marked differences in the preservational mineralogy.

Cretaceous forms are rare in all samples with the result that any given sample is difficult to date with confidence. However, the list of well-preserved and therefore identifiable specimens, taken as an aggregate for all the Site 460 samples, suggests that at least the Campanian Amphipyndax enesseffi Zone is represented. Some forms encountered here were not recognized at Site 452, where all the Cretaceous radiolarians are believed to belong to the $A$. enesseffi Zone, leaving open the possibility that other ages are represented at Site 460. As mentioned above, the poorly preserved (unidentifiable) material undoubtedly represents a different age.

The following list cites species identified and the sample in which they have been found. Bibliographic refer- 
ences are cited where they have not already been cited in the list for Site 452. Illustrations in this chapter are also cited.

Alievium sp.: Sample 460A-4-1, 70-72 cm

This chapter: Plate 4, Figure 1.

Amphipyndax enesseffi Foreman: Sample 460A-3-3, 70-72 cm. This chapter: Plate 4, Figures 2, 3.

Dictyomitra duodecimcostata duodecimcostata (Squinabol): Samples $460 \mathrm{~A}-3, \mathrm{CC} ; 460 \mathrm{~A}-4-1,70-72 \mathrm{~cm}$.

This chapter: Plate 4, Figure 6.

Dictyomitra spp.: Samples 460-1-4, 80-82 cm; 460A-2-4, 80-82 cm; $460 \mathrm{~A}-3-1,70-72 \mathrm{~cm}$; 460A-3,CC; 360A-4-1, 70-72 cm; 260A-5-1, $60-62 \mathrm{~cm}$.

This chapter: Plate 4, Figures 4, 5, 14.

Stichomitra asymbatos Foreman group(?): Sample 460A-4-1, 70$72 \mathrm{~cm}$.

This chapter: Plate 4, Figure 7.

Theocampe apicata Foreman, 1971, p. 1679, pl. 4, fig. 6: Sample $460 \mathrm{~A}-3, \mathrm{CC}$.

This chapter: Plate 4, Figure 8.

Theoperids gen. et. spp. indet.: Samples 460A-3-2, 70-72 cm; 460A$5-1,70-72 \mathrm{~cm}$.

Elongate ellipsoidal theoperids with distinct costae have been reported in several recent papers (e.g. Foreman, 1971; Kling, 1971; Moore, 1973) with incomplete systematics. Examples from Leg 60 materials exhibit considerable variability.

This chapter: Plate 4, Figure 9.

\section{Site $\mathbf{4 6 1}$}

As at Site 460 , both well and poorly preserved radiolarians occur at Site 461. And here too they are scarce and reworked into younger sediments.

The assemblage as a whole is dominated by cryptocapsid nassellarians almost all of which are unidentified. Therefore it remains impossible, as this is written, to date the Mesozoic radiolarians from Site 461. Diverse preservation again indicates heterogeneous sources and ages.

The following specimens have been identified or are illustrated to document the dominant forms present in the sample.

Actinommid gen. et. sp. indet.: Sample 461-2-4, 70-72 cm.

Note: Similar if not identical forms have been found by A. Schaaf (personal communication, 1979) in Leg 62 material.

This chapter: Plate 4, Figure 10.

Amphipyndax enesseffi Foreman: Sample 461-2,CC.

Amphipyndax sp.: Samples 461-1,CC, 461-2,CC.

This chapter: Plate 4, Figures 11, 12.

These forms are characterized by truncate conical post-thoracic segments with smooth, unornamented walls with small, diagonally aligned pores. The sutures are marked by elevated ridges.

Amphipyndax stocki (Campbell and Clark): Sample 461-1,CC.

Cryptamphorella conara Foreman), Dumitrică, 1970, p. 80, pl. 11, figs. 66a-c: Samples 461-1-1, 15-17 cm; 461-1,CC(?).

This chapter: Plate 5, Figure 4(?).

Dictyomitra sp.: Samples 461-1,CC, 461-2-3, 70-72 cm; 461-2-4, $70-72 \mathrm{~cm}$.

This chapter: Plate 4, Figure 14.

Gongylothorax verbeeki Foreman(?), p. 20, pl. 2, figs. 8a-c: Sample 461-1,CC.

Hemicryptocapsa simplex Dumitrică(?), p. 74, pl. 16, figs. 104a, b; pl. 21, figs. 142-148(?): Sample 461-2,CC.

Holocryptocapsa sp.: Sample 461-1,CC.

This chapter: Plate 5, Figures 7, 8.

Stichomitra asymbatos Foreman group: Sample 461-2-1, 70-72 cm. This chapter: Plate 4, Figure 13.

Theoperids gen. et. spp. indet.: Samples 461-1,CC; 461-2-4, 70$72 \mathrm{~cm}$.

Elongate ellipsoidal theoperids with distinct costae have been reported in a number of recent papers (e.g. Foreman, 1971; Kling, 1971; Moore, 1973) with incomplete systematics. The Site 461 mate- rials show an unusual amount of variability in pore arrangements and surface ornamentation.

This chapter: Plate 5, Figures 14-19.

\section{ACKNOWLEDGMENTS}

W. R. Riedel, A. Sanfilippo, A. Schaaf, and P. Baumgartner helped with identification of Mesozoic forms. D. L. Jones provided processing of the chert sample. C. Dean helped with preparation of samples. The manuscript was typed by G. Burns and reviewed by $C$. Nigrini, W. R. Riedel, and A. Sanfilippo. 1 am grateful to the Deep Sea Drilling Project for partial support of the work reported here.

\section{REFERENCES}

Bukry, D., Dinkelman, M. G., and Kaneps, A., 1973. Biostratigraphy of the equatorial East Pacific Rise. In van Andel, Tj. H., Heath, G. R., et al., Init. Repts. DSDP, 16: Washington (U.S. Govt. Printing Office), 915-935.

Dumitrică, P., 1970. Crytocephalic and cryptothoracic Nassellaria in some Mesozoic deposits of Romania. Rev. Roum. Géol. Geophys. Géogr., Sér. Géol., 14(1):45-124.

Foreman, H. P., 1966. Two Cretaceous radiolarian genera. Micropaleont., 12:355-359.

1968. Upper Maestrichtian Radiolaria of California. Paleont. Assoc. London, Special Paper 3.

1971. Cretaceous Radiolaria. In Winterer, E. L., Riedel, W. R., et al., Init. Repts. DSDP, 7, Pt. 2: Washington (U.S. Govt. Printing Office), 1673-1693.

, 1973. Radiolaria of Leg 10 with systematics and ranges for the families Amphipyndacidae, Artostrobiidae, and Theoperidae. In Worzel, J. L., Bryant, W., et al., Init. Repts. DSDP, 10: Washington (U.S. Govt. Printing Office), 407-474.

, 1975. Radiolaria from the North Pacific, Deep Sea Drilling Project Leg 32. In Larson, R. L., Moberly, R., et al., Init. Repts. DSDP, 32: Washington (U.S. Govt. Printing Office), 579-676. , 1977. Mesozoic Radiolaria from the Atlantic Basin and its borderlands. In Swain, F. M. (Ed.), Stratigraphic Micropaleontology of Atlantic Basin and Borderlands: Developments in Paleontology and Stratigraphy, 6: Amsterdam, Oxford, and New York (Elsevier), 305-320.

1978. Mesozoic Radiolaria in the Atlantic Ocean off the northwest coast of Africa, Deep Sea Drilling Project, Leg 41. In Lancelot, Y., Seibold, E., et al., Init. Repts. DSDP, 41: Washington (U.S. Govt. Printing Office), 739-761.

Friend, J. K., and Riedel, W. R., 1967. Cenozoic orosphaerid radiolarians from tropical Pacific sediments. Micropaleont., 13: 217-232.

Goll, R. M., 1968. Classification and phylogeny of Cenozoic Trissocyclidae (Radiolaria) in the Pacific and Caribbean Basin. Part 1. J. Paleont. 42:1409-1432.

Hardenbol, J., and Berggren, W. A., 1978. A new Paleogene numerical time scale. In Cohee, G. V., Glaessner, M. F., and Hedberg, H. D. (Eds.), Contributions to the Geologic Time Scale: Am. Assoc. Petrol. Geol., Studies in Geology, 6:213-234.

Johnson, D. A., and Knoll, A. H., 1975. Absolute ages of Quaternary radiolarian datum levels in the equatorial Pacific. Quaternary Res., 5:99-110.

Kling, S. A., 1971. Radiolaria, Leg 6 of the Deep Sea Drilling Project. In Fischer, A. G., Heezen, B. C., et al., Init. Repts. DSDP, 6: Washington (U.S. Govt. Printing Office), 1069-1117.

, 1973. Radiolaria from the eastern North Pacific, Deep Sea Drilling Project, Leg 18. In Kulm, L. D., von Huene, R., et al., Init. Repts. DSDP, 18: Washington (U.S. Govt. Printing Office), 617-671.

Knoll, A. H., and Johnson, D. A., 1975. Late Pleistocene evolution of the collosphaerid radiolarian Buccinosphaera invaginata Haeckel. Micropaleont., 21:60-68.

Moore, T. C., Jr., 1972 . Mid-Tertiary evolution of the radiolarian genus Calocycletta. Micropaleont., 18:144-152.

1973. Radiolaria from Leg 17 of the Deep Sea Drilling Project. In Winterer, E. L., Ewing, J. L., et al., Init. Repts. DSDP, 17: Washington (U.S. Govt. Printing Office), 797-869.

Nakaseko, K., 1955. Miocene radiolarian fossil assemblage from the southern Toyama Prefecture in Japan. Osaka Univ., Sci. Repts., 12:165-198. 
Nigrini, C., 1967. Radiolaria in pelagic sediments from the Indian and Atlantic Ocean. Calif. Univ., San Diego, Scripps Inst. Oceanography, Bull., 11:1-125.

, 1971. Radiolarian zones in the Quaternary of the equatorial Pacific Ocean. In Funnell, B. M. and Riedel, W. R. (Eds.), The Micropaleontology of Oceans: London (Cambridge University Press), 443-461.

1977. Tropical Cenozoic Artostrobiidae (Radiolaria). Micropaleont., 23:241-269.

Pessagno, E. A., Jr., 1963. Upper Cretaceous Radiolaria from Puerto Rico. Micropaleont., 9:197-214.

1972. Cretaceous Radiolaria, Part 1: The Phaseliformidae, new family, and other Spongodiscocea from the Upper Cretaceous portion of the Great Valley Sequence; Part 2: Pseudoaulophacidae Riedel from the Cretaceous of California and the Blake-Bahama Basin (JOIDES, Leg I). Bull. Am. Paleont., 61:267-328.

, 1976. Radiolarian zonation and stratigraphy of the Upper Cretaceous portion of the Great Valley Sequence, California Coast Ranges. Micropaleont., Special Publication, 2:1-95.

Pessagno, E. A., Jr., and Newport, R. L., 1972. A technique for extracting Radiolaria from radiolarian cherts. Micropaleont., 18: 231-234.

Riedel, W. R., 1957. Radiolaria: a preliminary stratigraphy. Swedish Deep-Sea Expedition 1947-1948, Repts., 6(3):59-96. , 1959. Oligocene and Lower Miocene Radiolaria in tropical Pacific sediments. Micropaleont., 5(3):285-302.

Riedel, W. R., and Sanfilippo, A., 1970. Radiolaria, Leg 4, Deep Sea Drilling Project. In Bader, R. G., Gerard, R. D., et al., Init.
Repts. DSDP, 4: Washington (U.S. Govt. Printing Office), 503-575.

, 1971. Cenozoic Radiolaria from the western tropical Pacific, Leg 7. In Winterer, E. L., Riedel, W. R., et al., Init. Repts. $D S D P, 7$, Pt. 2: Washington (U.S. Govt. Printing Office), 1529-1672.

, 1974. Radiolaria from the southern Indian Ocean, DSDP Leg 26. In Davies, T. A., Luyendyk, B. P., et al., Init. Repts. $D S D P, 26$ : Washington (U.S. Govt. Printing Office), 771-814. 1977. Cainozoic Radiolaria. In Ramsay, A. T. S. (Ed.), Oceanic Micropalaeont., 2: London, New York, and San Francisco (Academic Press), 847-912. 1978. Stratigraphy and evolution of tropical Cenozoic radiolarians. Micropaleont., 23:61-96.

Sanfilippo, A., and Riedel, W. R., 1970. Post-Eocene "closed" theoperid radiolarians. Micropaleont., 16(4):446-462.

1973. Cenozoic Radiolaria (exclusive of theoperids, artostrobiids, and amphipyndacids) from the Gulf of Mexico, DSDP Leg 10. In Worzel, J. L., Bryant, W., et al., Init. Repts. DSDP, 10: Washington (U.S. Govt. Printing Office), 475-611.

Theyer, F., Mato, C. Y., and Hammond, S. R., 1978. Paleomagnetic and geochronologic calibration of latest Oligocene to Pliocene radiolarian events, equatorial Pacific. Mar. Micropaleont., 3: 377-395.

Vincent, E., 1974. Cenozoic planktonic biostratigraphy and paleoceanography of the tropical western Indian Ocean. In Fischer, R. L., Bunce, E. T., et al., Init. Repts. DSDP, 24: Washington (U.S. Govt. Printing Office), 1111-1150. 


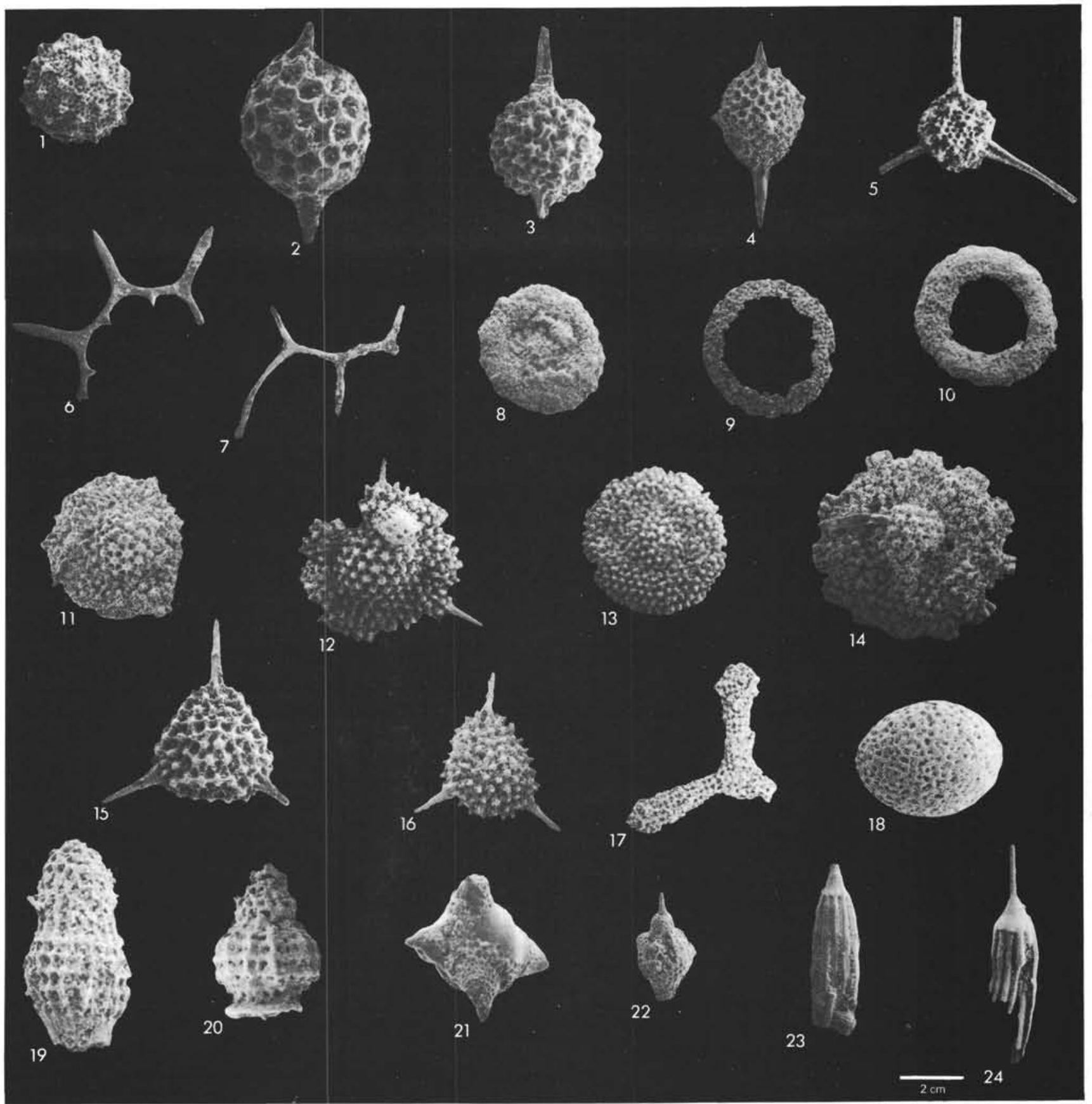

Plate 1. Cretaceous radiolarians. Bar scale $=100 \mu \mathrm{m}$, except Figures $2,19(=40 \mu \mathrm{m})$, Figure $20(=48 \mu \mathrm{m})$, and Figures $23,24(=200$ $\mu \mathrm{m})$. Magnification is $\times 100$, except Figures $2,19(\times 250)$, Figure $20(\times 210)$, and Figures 23, $24(\times 50)$.

Figures 1-4. Actinommids, gen. et spp. indet. Fig. 1, Sample $452-5$, CC. Figs. 2-4. Sample 452A-5-1, 28-30 cm.

Figure 5. Acaeniotyle sp. Sample 452A-5,CC.

Figures 6, 7. Spongosaturnalis(?) spp. Fig. 6. Sample 452A-5-1, $28-30 \mathrm{~cm}$. Fig. 7. Sample 452A-5,CC.

Figures 8-10. Spongodiscids, gen. et. spp. indet. Figs. 8, 10. Sample 452A-5-1, 28-30 cm. Fig. 9. Sample 452A-5,CC.

Figure 11. Pseudoaulophacus floresensis Pessagno(?). Sample 452A$5-1,28-30 \mathrm{~cm}$
Figure 12. Psendoaulophacus lenticulatus White(?). Sample 452A$5, \mathrm{CC}$.

Figures 13, 14. Pseudoaulophacus pargueraensis Pessagno. Fig. 13. Sample 452A-5,CC. Fig. 14. Sample 452A-5-1, 28-30 cm.

Figure 15. Alievium gallowayi (White). Sample 452A-5,CC.

Figure 16. Alievium gallowayi (White)(?). Sample 452A-5,CC.

Figure 17. Patulibracchium sp. Sample 452A-5,CC.

Figure 18. Phaseliforma sp. cf. P. laxa Pessagno. Sample 452A-5-1, $28-30 \mathrm{~cm}$.

Figure 19. Theocampe salillum Foreman. Sample 452A-5,CC.

Figure 20. Artostrobium urna Foreman. Sample 452A-5,CC.

Figure 21. Clathropyrgus titthium Riedel and Sanfilippo, ventrolateral view. Sample $452 \mathrm{~A}-5-1,28-30 \mathrm{~cm}$.

Figure 22. Podocapsa(?) sp. Sample 452A-5-1, 28-30 cm.

Figures 23, 24. Afens liriodes Riedel and Sanfilippo. Fig. 23. Sample 452A-5-1, 28-30 cm. Fig. 24. Sample 452A-5,CC. 


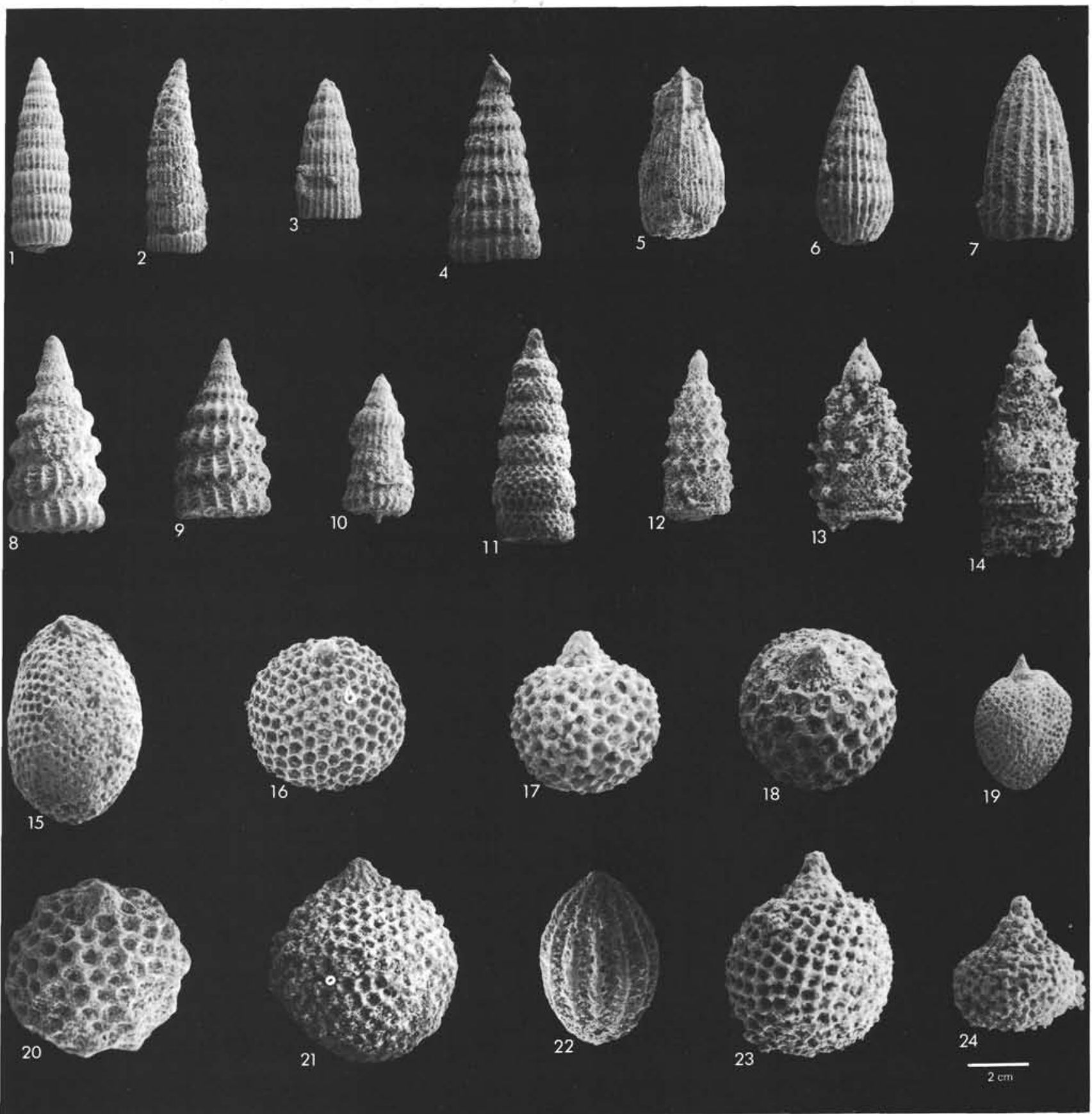

Plate 2. Cretaceous radiolarians. Bar scale $=100 \mu \mathrm{m}$ for Figures 1-6, $8-14,19 ;=40 \mu \mathrm{m}$ for Figures $15-18,20-24$. Magnification $\times 100$ for Figures 1-6, 8-14, 19; $\times 250$ for Figures 15-18, 20-24.

Figures 1-4. Dictyomitra spp. Figs. 1, 3. Sample 454-5-1, 28-30 cm. Figs. 2, 4. Sample 452A-5,CC.

Figure 5. Dictyomitra sp. X, Sample $452 \mathrm{~A}-5-1,28-30 \mathrm{~cm}$.

Figure 6. Archaeodictyomitra(?) sp. Sample 452A-5,CC.

Figure 7. Archaeodictyomitra sp. Sample $452 \mathrm{~A}-5-1,28-30 \mathrm{~cm}$.

Figures 8, 9. Dictyomitra duodecimcostata duodecimcostata (Squinabol), Sample 452A-5,CC.

Figure 10. Dictyomitra koslovae Foreman. Sample 452A-5,CC.
Figure 11. Amphipyndax stocki (Campbell and Clark). Sample 452A5-1, $28-30 \mathrm{~cm}$

Figure 12. Amphipyndax enesseffi Foreman. Sample 452A-5-1, 28$30 \mathrm{~cm}$.

Figures 13, 14. Stichomitra asymbatos Foreman group. Sample $452 \mathrm{~A}-5, \mathrm{CC}$.

Figure 15. Cryptocapsular theoperid(?) gen. et sp. indet. Sample $452 \mathrm{~A}-5-1,28-30 \mathrm{~cm}$.

Figure 16-19, 21-22, 24(?). Cryptocapsular theoperids, gen. et spp. indet. Figs. 16, 19, 22. Sample 452A-5-1, 28-30 cm. Figs. 17, 18, 21,24 . Sample $452 \mathrm{~A}-5, \mathrm{CC}$.

Figure 20. Hemicryptocapsa tuberosa Dumitrică(?). Sample 452A$5-1,28-30 \mathrm{~cm}$.

Figure 23. Theoperid, gen. et spp. indet. Sample 452A-5,CC. 


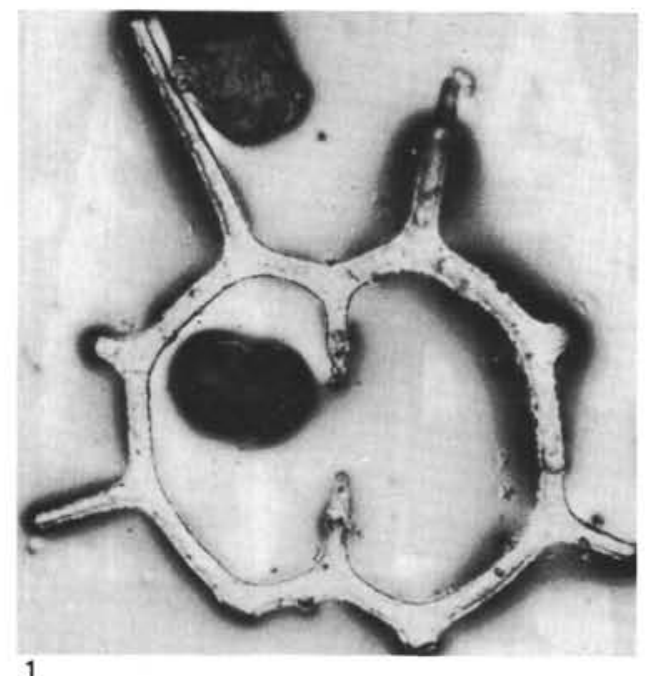

1

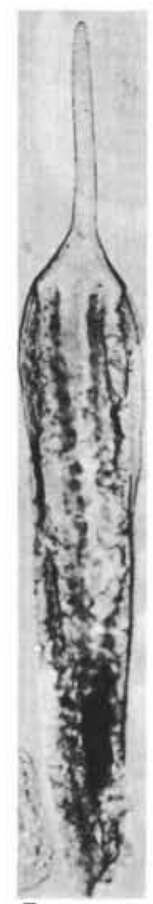

5

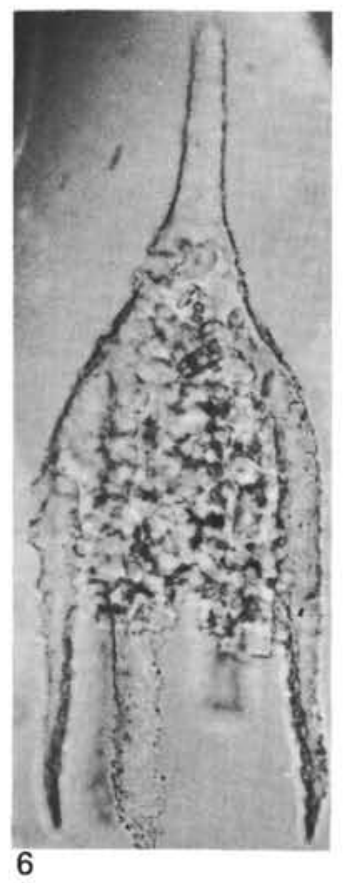

Plate 3. Cretaceous radiolarians. Bar scale $=100 \mu \mathrm{m}$, except Figure 1 $(=159 \mu \mathrm{m})$, Figure $5(=255 \mu \mathrm{m})$, and Figure $6(=125 \mu \mathrm{m})$. Magnification is $\times 265$, except Figure $1(\times 167)$, Figure $5(\times 104)$, and Figure $6(\times 212)$.

Figure 1-3. Spongosaturnalis (?) spp. Fig. 1. Sample 452A-5-1, 28-30 cm, sl. 1, P41/0. Fig. 2. Sample 452A-3-6, 20-22 cm, sl. 1, 059/3. Fig. 3. Sample $452 \mathrm{~A}-5-1,28-30 \mathrm{~cm}$, sl. 4 , D61/2.
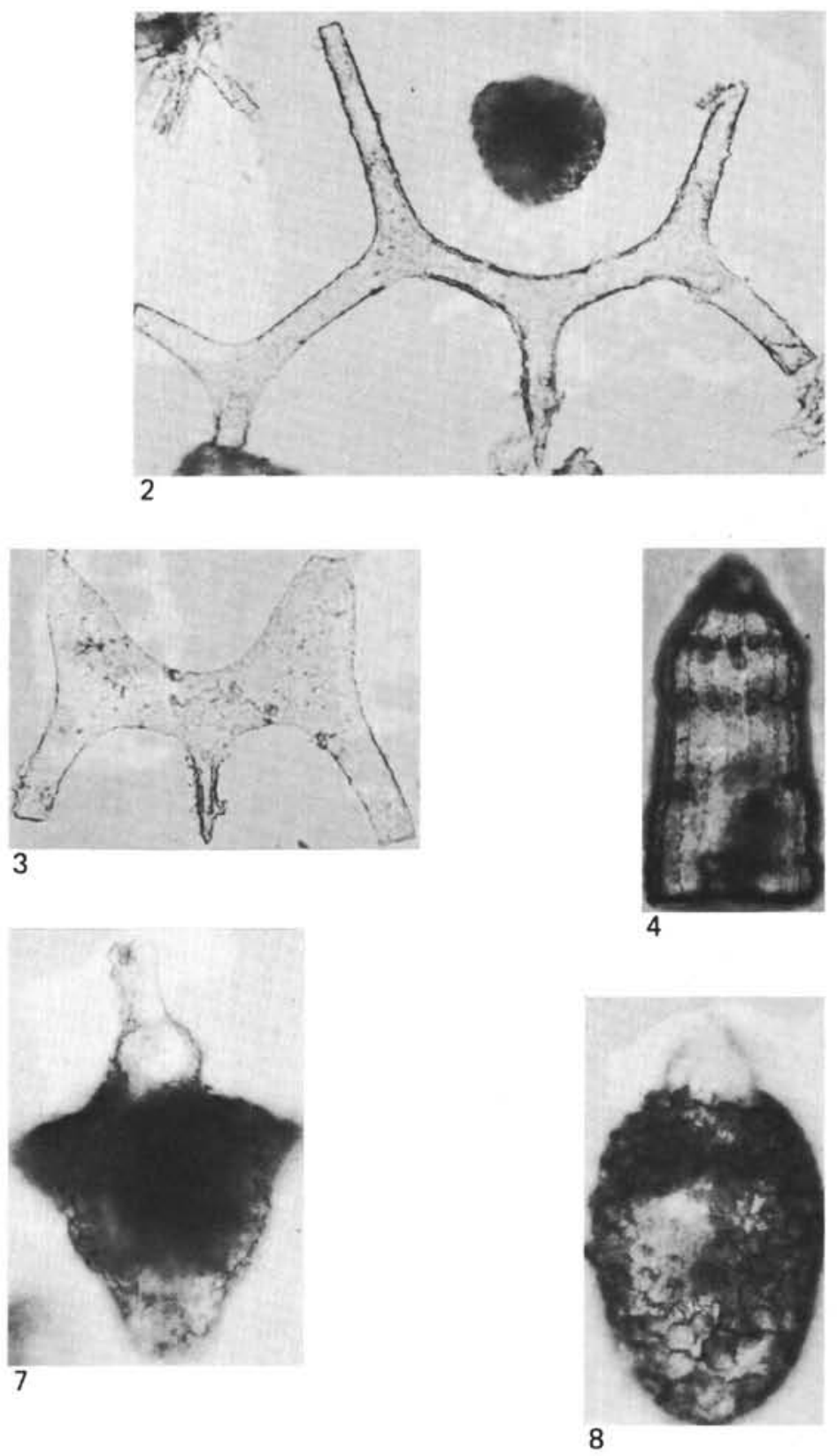

$2.65 \mathrm{~cm}$

Figure 4. Dictyomitra koslovae Foreman, Sample 452A-5-1, 28-30 $\mathrm{cm}, \mathrm{sl} .4, \mathrm{~J} 63 / 3$.

Figures 5, 6. Afens liriodes Riedel and Sanfilippo. Fig. 5. Sample 452A-3-4, 50-52 cm, sl. 1, D70/0. Fig. 6. Sample 452A-3-6, 20-22 $\mathrm{cm}$, sl. 1, A53/4.

Figure 7. Clathropyrgus titthium Riedel and Sanfilippo. Sample $452 \mathrm{~A}-3-6,20-22 \mathrm{~cm}$, sl. 2, P52/2.

Figure 8. Cryptocapsular theoperid(?), gen. et sp. indet. Sample $452 \mathrm{~A}-3-6,20-22 \mathrm{~cm}$, sl. $2, \mathrm{H} 66 / 0$. 

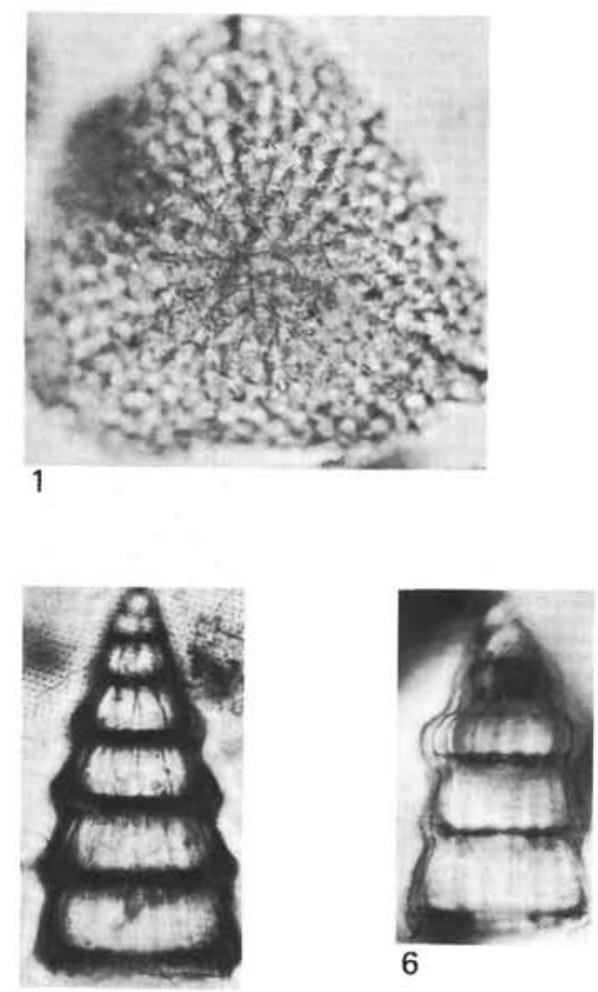

5
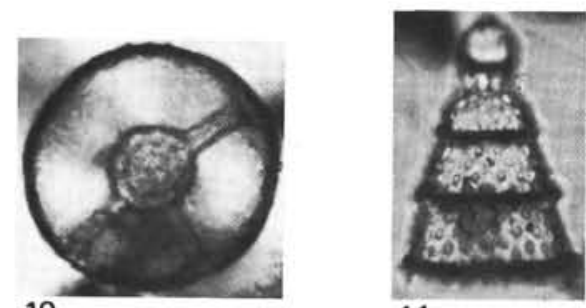

11

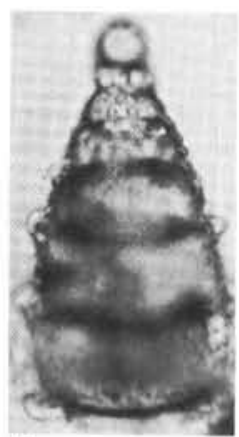

2
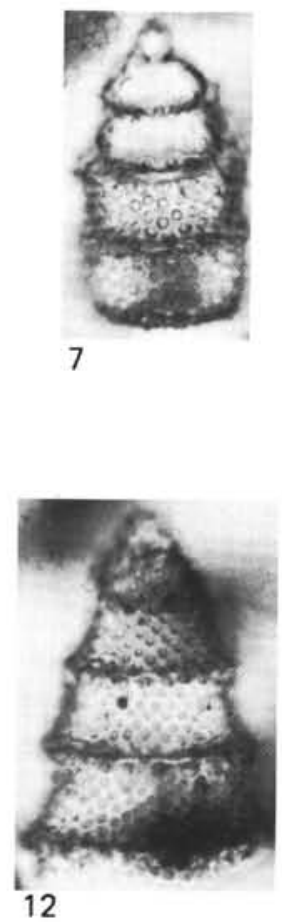

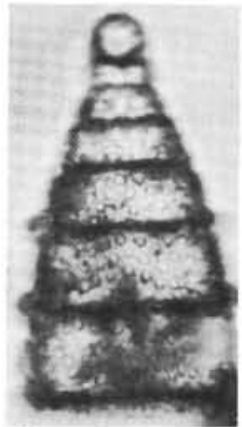

3
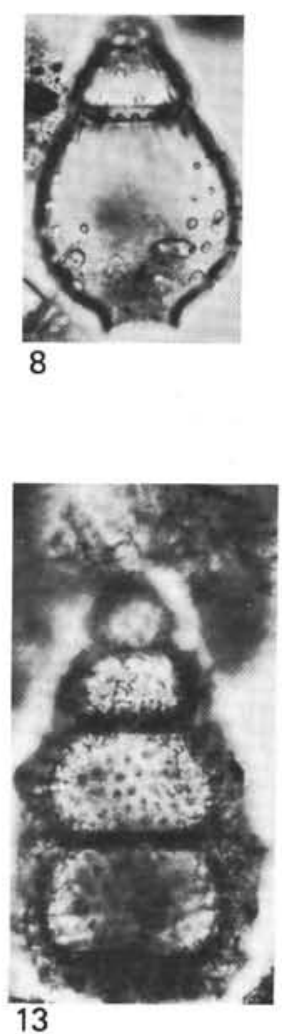

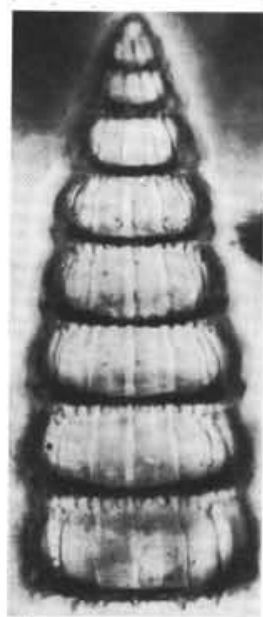

4

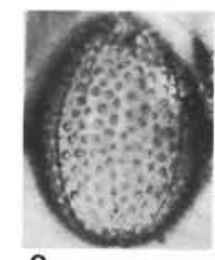

9

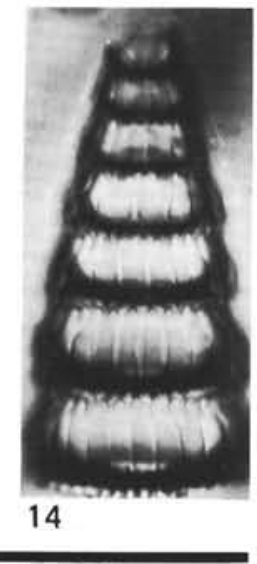

$2.65 \mathrm{~cm}$
Plate 4. Cretaceous radiolarians. Bar scale $=100 \mu \mathrm{m}$. Magnifications are $\times 265$.

Figure 1. Alievium sp. Sample 460A-4-1, 70-72 cm, sl. 1, X47/2.

Figures 2, 3. Amphipyndax enesseffi Foreman. Sample 460A-3-3, 70$72 \mathrm{~cm}$, sl. 2, B38/3.

Figures 4, 5. Dictyomitra spp. Fig. 4. Sample 460A-4-1, 70-72 cm, sl. 2, L69/4. Fig. 5. Sample 460-1-4, 80-82 cm, sl. 1, C60/0.

Figure 6. Dictyomitra duodecimcostata duodecimcostata (Squinabol). Sample 460A-4-1, 70-72 cm, sl. 1, R61/2.

Figure 7. Stichomitra asymbatos Foreman group(?). Sample 460A$4-1,70-72 \mathrm{~cm}, \mathrm{sl} .2$, V58/3.
Figure 8. Theocampe apicata Foreman. Sample 460A-3,CC, sl. 2, $\mathrm{J} 31 / 3$.

Figure 9. Theoperid, gen. et sp. indet. Sample 460A-5-1, 70-72 cm, sl. 2, Q54/0.

Figure 10. Actinommid, gen. et sp. indet. Sample 461-2-4, 70-72 cm, sl. $1, \mathrm{~N} 54 / 0$.

Figures 11, 12(?). Amphipyndax sp. Fig. 11. Sample 461-2,CC, sl. 2, C39/1. Fig. 12. Sample 461-1,CC, sl. 1, E34/0.

Figure 13. Stichomitra asymbatos Foreman group. Sample 461-2-1, $70-72 \mathrm{~cm}$, sl. 2 , H44/4.

Figure 14. Dictyomitra sp. Sample 461-2-3, 70-72 cm, sl. 2, S72/0. 


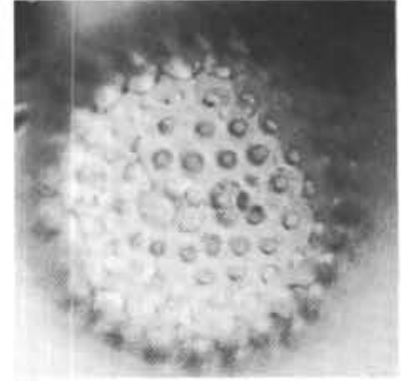

1

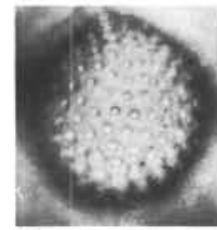

5

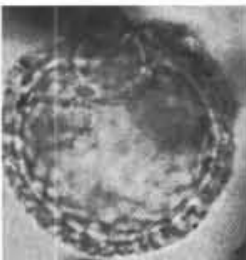

10

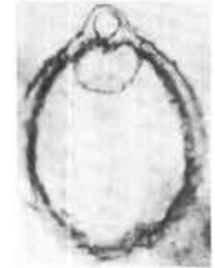

15

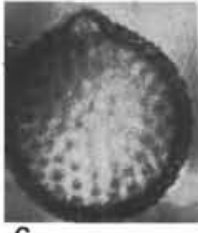

6

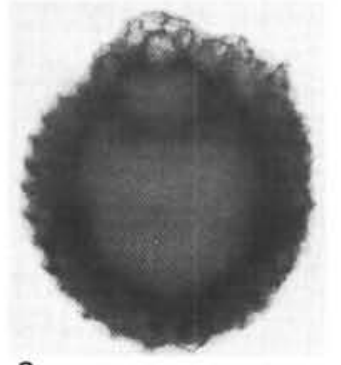

2

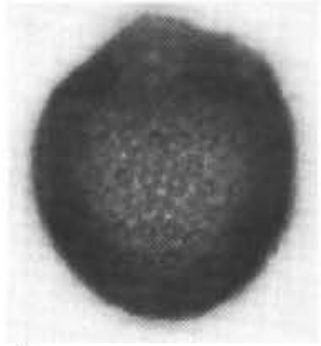

3
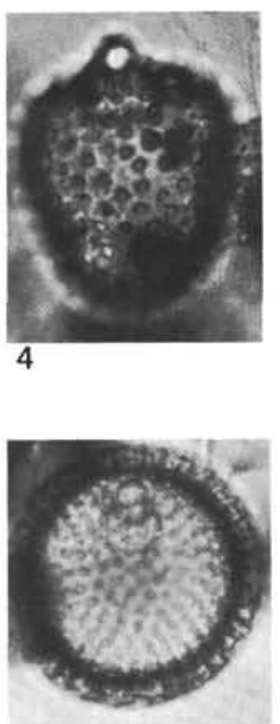

9

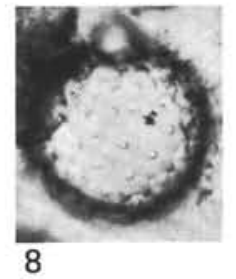

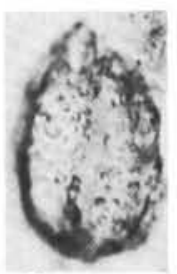

14

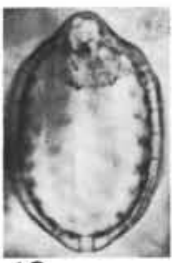

18

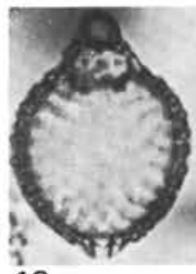

13

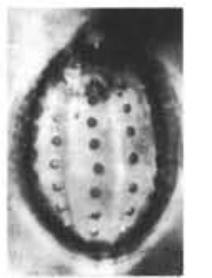

17

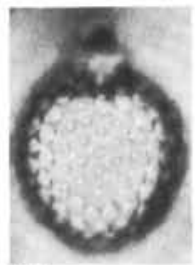

12

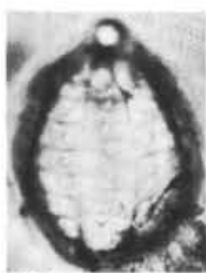

16
Plate 5. Cretaceous radiolarians. Bar scale $=100 \mu \mathrm{m}$, except Figure 1 $(=50 \mu \mathrm{m})$. Magnifications are $\times 265$, except Figure $1(\times 529)$.

Figures 1, 5, 6. Gongylothorax verbeeki Foreman. Sample 461-1,CC, sl. 2, F66/2. Fig. 1. Apical view showing collar pores and sutural pore.

Figures 2, 3, 9. Cryptocapsular theoperids, gen. et spp. indet. Figs. 2, 3. Sample 461-2-1, 70-72 cm, sl. 2, C57/1. Fig. 9. Sample 461-1,CC, sl. 2, B59/4.
Figure 4. Cryptamphorella conara Foreman(?). Sample 461-1,CC, sl. 2, G51/0.

Figures 7, 8. Holocryptocapsa sp. Sample 461-1,CC, sl. 2, W63/3. Figures 10, 11. Cryptamphorella conara Foreman. Sample 461-1-1, $15-17 \mathrm{~cm}$, sl. $1, \mathrm{~L} 44 / 3$.

Figures 12, 13. Hemicryptocapsa simplex Dumitrică(?). Sample 4612,CC, sl. 3, T30/0.

Figures 14-19. Theoperids, gen. et spp. indet. Fig. 14. Sample $461-2-4,70-72 \mathrm{~cm}$, sl. 1, M54/3. Figs. 15,16 . Sample 461-1,CC, sl. 2, D65/4. Fig. 17. Sample 461-1,CC, sl. 2, Q33/3. Figs. 18, 19. Sample 461-1,CC, sl. 2, N34/0. 